\title{
The rank of the semigroup of all order-preserving transformations on a finite fence
}

\author{
V.H. Fernandes, J. Koppitz and T. Musunthia ${ }^{\ddagger}$
}

October 21, 2019

\begin{abstract}
A zig-zag (or fence) order is a special partial order on a (finite) set. In this paper, we consider the semigroup $\mathcal{T F}_{n}$ of all order-preserving transformations on an $n$-element zig-zag ordered set. We determine the rank of $\mathcal{T F}_{n}$ and provide a minimal generating set for $\mathcal{T F}_{n}$. Moreover, a formula for the number of idempotents in $\mathcal{T F}_{n}$ is given.
\end{abstract}

\section{Introduction}

Let $n \in \mathbb{N}$ and denote by $\mathcal{T}_{n}$ the monoid (under composition) of all full transformations on the set $\bar{n}=\{1, \ldots, n\}$ of the first $n$ natural numbers. Let $\preceq$ be any partial order on $\bar{n}$. Let $\alpha \in \mathcal{T}_{n}$. We say that $\alpha$ is an order-preserving transformation (with respect to $\preceq$ ) if $x \preceq y$ implies $x \alpha \preceq y \alpha$, for all $x, y \in \bar{n}$. Clearly, the subset of $\mathcal{T}_{n}$ of all order-preserving transformations (with respect to a fixed partial order) forms a submonoid of $\mathcal{T}_{n}$.

A very important particular and natural case occurs when a linear order (for instance the one induced by the usual order on the natural numbers) is considered. The monoid $\mathcal{O}_{n}$ of all order-preserving transformations on $\bar{n}$, endowed with a linear order, has been extensively studied since the early 1960s. In fact, in 1962, Aǐzenštat $[1,2]$ showed that all non-trivial congruences of $\mathcal{O}_{n}$ are Rees congruences and gave a monoid presentation for $\mathcal{O}_{n}$, in terms of $2 n-2$ idempotent generators, from which it can be deduced that, for $n>1$, $\mathcal{O}_{n}$ only has one non-trivial automorphism. In 1971, Howie [13] calculated the cardinal and the number of idempotents of $\mathcal{O}_{n}$ and later (1992), jointly with Gomes [11], determined its rank and idempotent rank. More recently, Fernandes et al. [9] described the endomorphisms of the semigroup $\mathcal{O}_{n}$ by showing that there are three types of endomorphism: automorphisms, constants, and a certain type of endomorphism with two idempotents in the image. The monoid $\mathcal{O}_{n}$ also played a main role in several other papers $[3,7,8,10,12,16,17,19]$, where the central topic concerns the problem of the decidability of the pseudovariety generated by the family $\left\{\mathcal{O}_{n} \mid n \in \mathbb{N}\right\}$. This question was posed by J.-E. Pin in 1987 in the "Szeged International Semigroup Colloquium" and, as far as we know, is still open.

A non-linear order (in some sense) close to a linear order is the so-called zig-zag order. The pair $(\bar{n}, \preceq)$ is

${ }^{*}$ This work was developed within the FCT Project UID/MAT/00297/2013 of CMA and of Departamento de Matemática da Faculdade de Ciências e Tecnologia da Universidade Nova de Lisboa.

${ }^{\dagger}$ This work was developed within the FCT Project UID/MAT/00297/2013 of CMA.

${ }^{\ddagger}$ This work is also supported by Research Fund of Faculty of Science, Silpakorn University through Grant. no. SRF-PRG-2557-02. 2010 Mathematics Subject Classification: 20M10, 20M17, 20M20

Key words: Transformation semigroups, rank of semigroup, idempotents, order preserving, fence, zig-zag order 
called a zig-zag poset or fence if

$$
\begin{aligned}
& 1 \prec 2 \succ 3 \prec \cdots \prec n-1 \succ n, \quad \text { if } n \text { is odd, and } \\
& 1 \prec 2 \succ 3 \prec \cdots \succ n-1 \prec n, \quad \text { if } n \text { is even, or dually } \\
& 1 \succ 2 \prec 3 \succ \cdots \succ n-1 \prec n, \quad \text { if } n \text { is odd, and } \\
& 1 \succ 2 \prec 3 \succ \cdots \prec n-1 \succ n, \quad \text { if } n \text { is even. }
\end{aligned}
$$

The definition of the partial order $\preceq$ is self-explanatory. For instance, for $n=5$ and $n=6$, we have the following fences (given by Hasse diagrams):
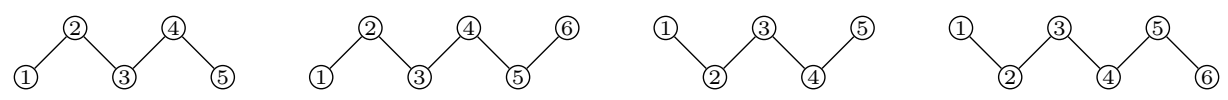

Observe that, every element in a fence is either minimal or maximal.

Order-preserving transformations of (finite) fences were first investigated by Currie and Visentin [5] and by Rutkowski [18]. In [5], by using generating functions, the authors calculate the number of order-preserving transformations of a fence with an even number of elements. On the other hand, an exact formula for the number of such transformations, for any natural number $n$, was given in [18].

Recently, several properties of monoids of order-preserving transformations of a fence were studied. In [4] the authors discussed the regular elements in these monoids. So-called coregular elements of this monoids were determined in [15]. On the other hand, in [6] Dimitrova and Koppitz investigated the monoid of all partial permutations preserving a zig-zag order on a set with $n$ elements, by studing Green's relations and generating sets of this monoid.

Without loss of generality, we will assume that $(\bar{n}, \preceq)$ is an up-fence, i.e.

$$
1 \prec 2 \succ 3 \prec \cdots \text {. }
$$

Let $x, y \in \bar{n}$. We say that $x$ and $y$ are comparable if $x \prec y$ or $x=y$ or $y \prec x$. Otherwise, $x$ and $y$ are said incomparable. Clearly, $x$ and $y$ are comparable if and only if $x \in\{y-1, y, y+1\}$.

Denote by $\mathcal{T F}_{n}$ the submonoid of $\mathcal{T}_{n}$ of all order-preserving transformations of the fence $(\bar{n}, \preceq)$.

In this paper, we determine the rank and count the number of idempotents of $\mathcal{T F}_{n}$.

Recall that the rank of a (finite) semigroup $S$ is defined by

$$
\operatorname{rank} S=\min \{|A| \mid A \subseteq S \text { generates } S\},
$$

i.e. the rank of $S$ is the minimal size of a generating set of $S$. For general background on Semigroup Theory and standard notation, we refer the reader to Howie's book [14].

We begin, in the next section, by giving a characterization of the elements of $\mathcal{T F}_{n}$. Clearly, the identity mapping $\mathrm{id}_{n}$ on $\bar{n}$ is order-preserving. Also, all the $n$ constant mappings are order-preserving. Moreover, for an even $n, \mathrm{id}_{n}$ is the unique permutation of $\bar{n}$ belonging to $\mathcal{T F}_{n}$ and, on the other hand, if $n$ is odd then $\mathcal{T F}_{n}$ has exactly two permuations, namely the identity mapping and the reflection

$$
\gamma_{n}=\left(\begin{array}{cccc}
1 & 2 & \cdots & n \\
n & n-1 & \cdots & 1
\end{array}\right)
$$

The rest of Section 2 is dedicated to counting the idempotents of $\mathcal{T F}_{n}$. Notice that, it is easy to show that an element $\alpha \in \mathcal{T}_{n}$ is idempotent if and only if $\operatorname{Im} \alpha=\{x \in \bar{n} \mid x \alpha=x\}$, i.e. the image of $\alpha$ coincides with the set of its fix points. In the third section of this paper, we determine the rank of $\mathcal{T F}_{n}$. In particular, we provide a minimal size generating set for $\mathcal{T} \mathcal{F}_{n}$.

Notice that $\mathcal{T F}_{1}$ coincides with $\mathcal{T}_{1}$ and $\mathcal{T F}_{2}$ coincides with the monoid $\mathcal{O}_{2}$ of all order-preserving transformations on a two-element chain. Hence, from now on, we always consider $n \geq 3$. 


\section{Idempotents}

The aim of this section is to provide a formula for the number of idempotents of $\mathcal{T} \mathcal{F}_{n}$. In order to accomplish this, it is useful to know the form of the elements of $\mathcal{T F}_{n}$. We have the following characterization of a transformation in $\mathcal{T F}_{n}$.

Theorem 2.1. Let $\alpha \in \mathcal{T}_{n}$. Then $\alpha \in \mathcal{T F}_{n}$ if and only if

(i) $|x \alpha-(x+1) \alpha| \leq 1$, for all $x \in\{1, \ldots, n-1\}$;

(ii) $x$ and $x \alpha$ have the same parity or $(x-1) \alpha=x \alpha=(x+1) \alpha$, for all $x \in\{2, \ldots, n-1\}$.

Proof. First, suppose that $\alpha \in \mathcal{T F}_{n}$. Let $x \in\{1, \ldots, n-1\}$. Then $x$ and $x+1$ are comparable, which implies that $x \alpha$ and $(x+1) \alpha$ are also comparable and so $|x \alpha-(x+1) \alpha| \leq 1$. This shows (i). Now let $x \in\{2, \ldots, n-1\}$. Assume that $x$ is even. Then $x-1 \prec x \succ x+1$ and so $(x-1) \alpha \preceq x \alpha \succeq(x+1) \alpha$. If $(x-1) \alpha \neq x \alpha$ or $x \alpha \neq(x+1) \alpha$ then $(x-1) \alpha \prec x \alpha$ or $x \alpha \succ(x+1) \alpha$, which implies in both cases that $x \alpha$ is even. Similarly, if $x$ is odd we may deduce that $x \alpha$ is also odd or $(x-1) \alpha=x \alpha=(x+1) \alpha$. This shows (ii).

Conversely, suppose that (i) and (ii) are satisfied. Let $x, y \in \bar{n}$ be such that $x \prec y$. Then $x$ is odd and $y$ is even. Moreover $y \in\{x-1, x+1\}$. Admit that $x \alpha \neq y \alpha$. If $y=x-1$ then $2 \leq y \leq n-1$ and so $|y \alpha-x \alpha|=|y \alpha-(y+1) \alpha|=1$ and $y$ and $y \alpha$ have the same parity. If $y=x+1$ then $1 \leq x \leq n-1$ and so $|x \alpha-y \alpha|=|x \alpha-(x+1) \alpha|=1$. Furthermore, in this last case, if $x>1$ then $x$ and $x \alpha$ have the same parity; otherwise $y=2<n$ and so $y$ and $y \alpha$ have the same parity ( $\operatorname{since}(y-1) \alpha=x \alpha \neq y \alpha$ ). Therefore, we have $y \alpha \in\{x \alpha-1, x \alpha+1\}$ and, on the other hand, $y \alpha$ is even or $x \alpha$ is odd. Thus, in all cases, $x \alpha \prec y \alpha$, as required.

As a consequence of Theorem 2.1 we have that the image of a transformation in $\mathcal{T F}_{n}$ is an interval of $\bar{n}$ (with the usual order).

Corollary 2.2. Let $\alpha \in \mathcal{T F}_{n}$. Then $\operatorname{Im} \alpha=\{k, k+1, \ldots, \ell\}$, for some $1 \leq k<\ell \leq n$.

Proof. Let $k=\min \operatorname{Im} \alpha$ and $\ell=\max \operatorname{Im} \alpha$ (with respect to the usual order of $\mathbb{N}$ ). Assume that there exists $p \in\{k, k+1, \ldots, \ell\}$ such that $p \notin \operatorname{Im} \alpha$. Let $x=\max \{i \in \bar{n} \mid i \alpha<p\}$. If $x<n$ then $(x+1) \alpha>p$ and so $|x \alpha-(x+1) \alpha|>1$, a contradiction. Then $y=\max \{i \in \bar{n} \mid i \alpha>p\}<n$ and $(y+1) \alpha<p$, whence $|y \alpha-(y+1) \alpha|>1$, which again is a contradiction. Thus $\operatorname{Im} \alpha=\{k, k+1, \ldots, \ell\}$, as required.

Next we will give a formula for the number of idempotents in $\mathcal{T F}_{n}$. Let $m \in \bar{n}$ and $0 \leq p \leq n-m$. For $r \in\{0, \ldots, m-1\}$, let

$$
P(p, r)=\left\{\left(p_{0}, \ldots, p_{t}\right) \mid t \in \mathbb{N} \cup\{0\} ; p_{1}, \ldots, p_{t} \in \mathbb{N} ; p_{0}=0 ; 0 \leq \sum_{i=1}^{s}(-1)^{i+1} p_{i} \leq p, \text { for } 1 \leq s \leq t ; \sum_{i=1}^{t} p_{i}=r\right\}
$$

and

$$
K(m, r)=\left\{\left(k_{0}, \ldots, k_{r}\right) \mid k_{0}+r+2 \sum_{i=1}^{r} k_{i}=m-1, k_{0}, \ldots, k_{r} \in \mathbb{N} \cup\{0\}\right\}
$$

Further, define

$$
A(m, p)=\sum_{r=0}^{m-1}|P(p, r)| \cdot|K(m, r)| .
$$

Lemma 2.3. Let $\alpha \in \mathcal{T F}_{n}$ with $\operatorname{Im} \alpha=\{k, \ldots, k+p\}$, for some $k \in \bar{n}$ and some $p \in\{0, \ldots, n-k\}$. Let $a_{0} \in\{k, k+p\}$ and $r \in\{0, \ldots, k-1\}$. Then, there exists a bijection between the set $P(p, r)$ and the set of all sequences $a_{0}, a_{1}, \ldots, a_{r} \in \operatorname{Im} \alpha$ such that $\left|a_{i-1}-a_{i}\right|=1$, for all $i \in\{1, \ldots, r\}$, and there exists a partition $A_{0}>A_{1}>\cdots>A_{r}$ of $\{1, \ldots, k\}$, if $a_{0}=k$, or a partition $A_{0}<A_{1}<\cdots<A_{r}$ of $\{k+p, \ldots, n\}$, if $a_{0}=k+p$, verifying $A_{i} \alpha=\left\{a_{i}\right\}$, for $i \in\{0, \ldots, r\}$. 
Proof. Fix a sequence $a_{0}, a_{1}, \ldots, a_{r} \in \operatorname{Im} \alpha$ verifying the conditions of the lemma. Notice that, if $r=0$ then $P(p, 0)=\{(0)\}$ and $a_{0}$ is the only possible sequence. Then, we may admit that $r>0$. Let $j=1$, if $a_{0}=k$, or $j=2$, if $a_{0}=k+p$. Put $p_{0}=0$ (by technical reasons).

Then, there exists $p_{1} \in\{1, \ldots, r\}$ such that $(-1)^{1+1} p_{1} \in\{0, \ldots, p\}, a_{i}=a_{0}+(-1)^{j+1} i$, for $1 \leq i \leq p_{1}$, and either $r=p_{1}$ or $a_{p_{1}+1}=a_{0}+(-1)^{j+1} p_{1}+(-1)^{j+2}$.

If $r>p_{1}$ then there exists $p_{2} \in\left\{1, \ldots, r-p_{1}\right\}$ such that $(-1)^{1+1} p_{1}+(-1)^{2+1} p_{2} \in\{0, \ldots, p\}, a_{p_{1}+i}=a_{0}+$ $(-1)^{j+1} p_{1}+(-1)^{j+2} i$, for $1 \leq i \leq p_{2}$, and either $r=p_{1}+p_{2}$ or $a_{p_{1}+p_{2}+1}=a_{0}+(-1)^{j+1} p_{1}+(-1)^{j+2} p_{2}+(-1)^{j+3}$.

Continuing in this way, we obtain $t, p_{1} \ldots, p_{t} \in \mathbb{N}$ such that

$$
\sum_{i=1}^{t} p_{i}=r, \quad \sum_{i=1}^{s}(-1)^{i+1} p_{i} \in\{0, \ldots, p\}, \quad \text { for } 1 \leq s \leq t,
$$

and

$$
a_{i+\sum_{\ell=1}^{q-1} p_{\ell}}=a_{0}+\sum_{\ell=1}^{q-1}(-1)^{j+\ell} p_{\ell}+(-1)^{j+q} i, \quad \text { for } 1 \leq i \leq p_{q} \text { and } 1 \leq q \leq t .
$$

Hence, the sequence $a_{0}, a_{1}, \ldots, a_{r}$ is uniquely determined by the $t$-uple $\left(p_{0}, \ldots, p_{t}\right)$.

Let us denote by $E_{m}$ the set of all idempotents of $\mathcal{T F}_{m}$, for all $m \geq 1$. It is clear that $E_{1}=\mathcal{T F}_{1}=\mathcal{T}_{1}=\left\{\left(\begin{array}{l}1 \\ 1\end{array}\right)\right\}$ and $E_{2}=\mathcal{T F}_{2}=\mathcal{T}_{2} \backslash\left\{\left(\begin{array}{l}12 \\ 21\end{array}\right)\right\}=\left\{\left(\begin{array}{l}12 \\ 12\end{array}\right),\left(\begin{array}{l}12 \\ 11\end{array}\right),\left(\begin{array}{c}12 \\ 22\end{array}\right)\right\}$.

Theorem 2.4. We have

$$
\left|E_{n}\right|=\sum_{k=1}^{n} \sum_{p=0}^{n-k} A(k, p) \cdot A(n+1-(k+p), p) .
$$

Proof. Let $\alpha \in E_{n}$. Then, by Corollary 2.2, there exist $k \in \bar{n}$ and $p \in\{0, \ldots, n-k\}$ such that

$$
\operatorname{Im} \alpha=\{k, k+1, \ldots, k+p\} .
$$

Since $\alpha$ is idempotent, we have $(k+i) \alpha=k+i$, for $i \in\{0, \ldots, p\}$. Let

$$
A^{-}=\{1, \ldots, k\} \quad \text { and } \quad A^{+}=\{k+p, \ldots, n\} .
$$

First, we consider the set $A^{-}$. By Theorem 2.1, we have $|x \alpha-(x+1) \alpha| \leq 1$ for all $x \in\{1, \ldots, k-1\}$. Hence, there exist $r \in\{0, \ldots, k-1\}$, a sequence $a_{0}, \ldots, a_{r} \in \operatorname{Im} \alpha$ and a partition $A_{0}>A_{1}>\cdots>A_{r}$ of $A^{-}$ such that $\left|a_{i-1}-a_{i}\right|=1$, for $1 \leq i<r$, and $A_{i} \alpha=\left\{a_{i}\right\}$, for $0 \leq i \leq r$. Moreover, $x \alpha$ and $x$ have the same parity or $(x-1) \alpha=x \alpha=(x+1) \alpha$, for all $x \in A^{-} \backslash\{1, n\}$. It follows that there exist $k_{0}, k_{1}, \ldots, k_{r} \in \mathbb{N} \cup\{0\}$ such that $\left|A_{i}\right|=1+2 k_{i}$, for $0 \leq i \leq r-1$, and $\left|A_{r}\right|=k_{r}+1$. Then $k_{r}+r+2 \sum_{i=0}^{r-1} k_{i}=k-1$ and so the sequence $A_{0}>A_{1}>\cdots>A_{r}$ is uniquely determined by an element of $K(k, r)$.

If $r=0$ then $A^{-}=A_{0}$ and $P(p, 0)=\{(0)\}$. On the other hand, admit that $r>0$. Then, by Lemma 2.3 (with $a_{0}=k$ ), we have that the sequence $a_{0}, \ldots, a_{r}$ is uniquely determined by an element of the set $P(p, r)$. Hence, $\left.\alpha\right|_{A^{-}}$is uniquely determined by an element of the set

$$
B^{-}(k, p)=\bigcup_{r=0}^{k-1} K(k, r) \times P(p, r) \times\{r\} .
$$

Dually, there exist $s \in\{0, \ldots, n-(k+p)\}$, a sequence $a_{0}, \ldots, a_{s} \in \operatorname{Im} \alpha$ and a partition $A_{0}<A_{1}<\cdots<A_{s}$ of $A^{+}$such that $\left|a_{i-1}-a_{i}\right|=1$, for $1 \leq i<s$, and $A_{i} \alpha=\left\{a_{i}\right\}$, for $0 \leq i \leq s$. Also, there exist $\ell_{0}, \ell_{1}, \ldots, \ell_{s} \in \mathbb{N} \cup\{0\}$ such that $\left|A_{i}\right|=1+2 \ell_{i}$, for $0 \leq i \leq s-1$, and $\left|A_{s}\right|=\ell_{s}+1$. Then $\ell_{r}+r+2 \sum_{i=0}^{s-1} \ell_{i}=n-(k+p)=(n+1)-(k+p)-1$, whence the sequence $A_{0}<A_{1}<\cdots<A_{s}$ is uniquely determined by an element of $K(n+1-(k+p), s)$. 
If $s=0$ then $A^{+}=A_{0}$ and $P(p, 0)=\{(0)\}$. So, admit that $s>0$. Then, by Lemma 2.3 (with $\left.a_{0}=k+p\right)$, we have that the sequence $a_{0}, \ldots, a_{s}$ is uniquely determined by an element of the set $P(p, s)$. Consequently, $\left.\alpha\right|_{A^{+}}$is uniquely determined by an element of the set

$$
B^{+}(k, p)=\bigcup_{s=0}^{n-(k+p)} K(n+1-(k+p), s) \times P(p, s) \times\{s\} .
$$

Notice that, it is easy to verify that $\left|B^{-}(k, p)\right|=A(k, p)$ and $\left|B^{+}(k, p)\right|=A(n+1-(k+p), p)$. Moreover, $\left.\alpha\right|_{\operatorname{Im} \alpha}$ is the identity mapping on $\operatorname{Im} \alpha$ and $\operatorname{Im} \alpha$ is uniquely determined by an element $k$ of the set $\bar{n}$ and an element $p$ of the set $\{0, \ldots, n-k\}$. Thus, the transformation $\alpha \in E_{n}$ is uniquely determined by an element of the set

$$
\bigcup_{k=1}^{n} \bigcup_{p=0}^{n-k} B^{-}(k, p) \times B^{+}(k, p) \times\{(k, p)\} .
$$

Conversely, as the construction of this set clearly justifies that each of its elements determines uniquely an idempotent in $\mathcal{T F}_{n}$, we have

$$
\begin{aligned}
\left|E_{n}\right| & =\left|\bigcup_{k=1}^{n} \bigcup_{p=0}^{n-k} B^{-}(k, p) \times B^{+}(k, p) \times\{(k, p)\}\right|=\sum_{k=1}^{n} \sum_{p=0}^{n-k}\left|B^{-}(k, p) \times B^{+}(k, p) \times\{(k, p)\}\right| \\
& \left.=\sum_{k=1}^{n} \sum_{p=0}^{n-k}\left|B^{-}(k, p)\right| \cdot \mid B^{+}(k, p)\right\}|\cdot|\{(k, p)\} \mid=\sum_{k=1}^{n} \sum_{p=0}^{n-k} A(k, p) \cdot A(n+1-(k+p), p),
\end{aligned}
$$

as required.

The table below gives us an idea of the size of the monoids $\mathcal{T F}_{m}$ and of their number of idempotents.

\begin{tabular}{|c|c|c|}
\hline$m$ & $\left|E_{m}\right|$ & $\left|\mathcal{T F}_{m}\right|$ \\
\hline 1 & 1 & 1 \\
\hline 2 & 3 & 3 \\
\hline 3 & 8 & 11 \\
\hline 4 & 19 & 31 \\
\hline 5 & 44 & 99 \\
\hline 6 & 98 & 275 \\
\hline 7 & 218 & 811 \\
\hline 8 & 474 & 2199 \\
\hline
\end{tabular}

\begin{tabular}{|c|c|c|}
\hline$m$ & $\left|E_{m}\right|$ & $\left|\mathcal{T F}_{m}\right|$ \\
\hline 9 & 1039 & 6187 \\
\hline 10 & 2243 & 16459 \\
\hline 11 & 4901 & 44931 \\
\hline 12 & 10591 & 117831 \\
\hline 13 & 23190 & 315067 \\
\hline 14 & 50335 & 817323 \\
\hline 15 & 110651 & 2152915 \\
\hline 16 & 241457 & 5537839 \\
\hline
\end{tabular}

These numbers were calculated by the formula of Theorem 2.4 and by the formulas given by Rutkowski [18].

\section{The rank of $\mathcal{T F}_{n}$}

This section is devoted to determine the rank of $\mathcal{T F}_{n}$. In the process we give an explicit minimal size set of generators of $\mathcal{T F}_{n}$. The cases $n$ odd and $n$ even will be treated separately.

The following general observation will be frequently used without reference.

Lemma 3.1. Let $\alpha, \alpha^{\prime} \in \mathcal{T F}_{n}$ be such that $\operatorname{Ker} \alpha=\operatorname{Ker} \alpha^{\prime}$ and $\operatorname{rank} \alpha>1$. Then $x \alpha$ and $x \alpha^{\prime}$ have the same parity, for all $x \in \bar{n}$.

Proof. Let $x \in \bar{n}$. Since $\operatorname{rank} \alpha>1$, there exists $y \in x \alpha \alpha^{-1}$ such that $y+1 \in \bar{n} \backslash y \alpha \alpha^{-1}$ or $y-1 \in \bar{n} \backslash y \alpha \alpha^{-1}$. Therefore we may consider four cases. For instance, if $y+1 \in \bar{n} \backslash y \alpha \alpha^{-1}$ and $y \prec y+1$ then $x \alpha=y \alpha \prec(y+1) \alpha$ and $x \alpha^{\prime}=y \alpha^{\prime} \prec(y+1) \alpha^{\prime}$, whence $x \alpha$ and $x \alpha^{\prime}$ have the same parity. The other three cases are similar. 
Next, we define a series of transformations of $\mathcal{T} \mathcal{F}_{n}$. Let (for any $n$ )

$$
\begin{aligned}
& \alpha_{1,2}=\left(\begin{array}{ccccc}
\overline{1,2} & 3 & 4 & \cdots & n \\
2 & 3 & 4 & \cdots & n
\end{array}\right)
\end{aligned}
$$

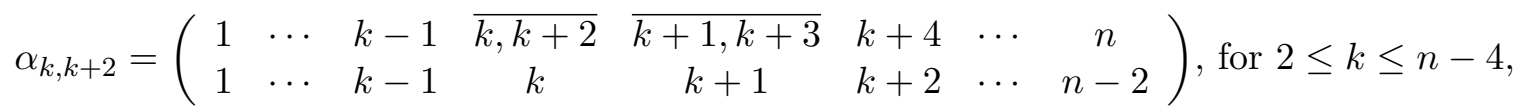

$$
\begin{aligned}
& \alpha_{n-2, n}=\left(\begin{array}{ccccc}
1 & \cdots & n-3 & \overline{n-2, n} & n-1 \\
1 & \cdots & n-3 & n-2 & n-1
\end{array}\right) \text {, for } n \geq 4 \text {, }
\end{aligned}
$$

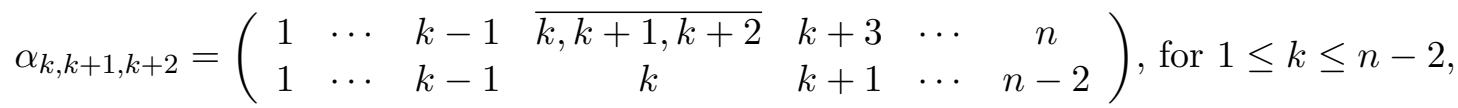

$$
\begin{aligned}
& \alpha_{1,2 k+1}=\left(\begin{array}{cccccccc}
k+1 & \overline{k, k+2} & \ldots & \overline{2,2 k} & \overline{1,2 k+1} & 2 k+2 & \cdots & n \\
k+1 & k+2 & \ldots & 2 k & 2 k+1 & 2 k+2 & \cdots & n
\end{array}\right) \text {, for } 1 \leq k \leq\left\lfloor\frac{n-1}{2}\right\rfloor \text {, and } \\
& \beta_{k, m}=\left(\begin{array}{cccccc}
1 & \cdots & k-1 & \overline{k, k+2 m} & \overline{k+1, k+2 m-1, k+2 m+1} & \cdots \\
1 & \cdots & k-1 & k & k+1 & \cdots
\end{array}\right.
\end{aligned}
$$

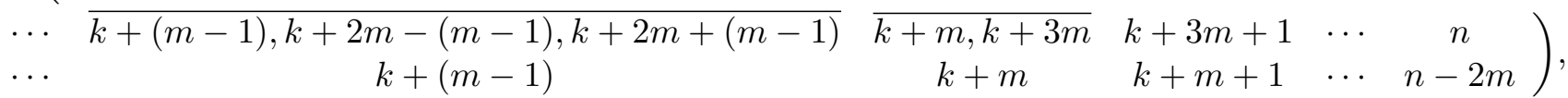

for $2 \leq k, m \leq n$ such that $k+3 m \leq n-1$.

Moreover, for an odd $n$, recall that

$$
\gamma_{n}=\left(\begin{array}{ccccc}
1 & 2 & \cdots & n-1 & n \\
n & n-1 & \cdots & 2 & 1
\end{array}\right)
$$

and, for an even $n$, let

$$
\begin{aligned}
& \alpha_{1,2}^{e}=\left(\begin{array}{ccccc}
\overline{1,2} & 3 & 4 & \cdots & n \\
n & n-1 & n-2 & \cdots & 2
\end{array}\right) \text {, } \\
& \alpha_{n-1, n}=\left(\begin{array}{ccccc}
1 & 2 & \cdots & n-2 & \overline{n-1, n} \\
n-1 & n-2 & \cdots & 2 & 1
\end{array}\right) \\
& \alpha_{2 k, n}=\left(\begin{array}{cccccccc}
1 & \cdots & 2 k-1 & \frac{n}{2}+k & \overline{\frac{n}{2}+k-1, \frac{n}{2}+k+1} & \overline{\frac{n}{2}+k-2, \frac{n}{2}+k+2} & \cdots & \overline{2 k, n} \\
1 & \cdots & 2 k-1 & \frac{n}{2}+k & \frac{n}{2}+k-1 & \frac{n}{2}+k-2 & \cdots & 2 k
\end{array}\right) \text {, }
\end{aligned}
$$

for $1 \leq k \leq \frac{n-4}{2}$, and

$$
\alpha_{1,2 k+1}^{e}=\left(\begin{array}{cccccccc}
k+1 & \overline{k, k+2} & \ldots & \overline{2,2 k} & \overline{1,2 k+1} & 2 k+2 & \cdots & n \\
k-1 & k & \cdots & 2 k-2 & 2 k-1 & 2 k & \cdots & n-2
\end{array}\right), \text { for } 2 \leq k \leq \frac{n-2}{2} .
$$

Now, for an odd $n$, define

$$
\begin{aligned}
G_{n}= & \left\{\gamma_{n}, \alpha_{1,2}\right\} \cup\left\{\alpha_{k, k+2} \mid 2 \leq k \leq \frac{n-3}{2}\right\} \cup\left\{\alpha_{k, k+1, k+2} \mid 1 \leq k \leq \frac{n-1}{2}\right\} \cup \\
& \left\{\alpha_{1,2 k+1} \mid 1 \leq k \leq \frac{n-1}{2}\right\} \cup\left\{\beta_{k, m} \mid 2 \leq k, m \leq \frac{n-1}{2} \text { and } 2 k+3 m \leq n+1\right\}
\end{aligned}
$$

and, for an even $n$, define

$$
\begin{aligned}
G_{n}= & \left\{\operatorname{id}_{n}, \alpha_{1,2}^{e}, \alpha_{1,3}, \alpha_{n-1, n}, \alpha_{n-2, n}\right\} \cup\left\{\alpha_{k, k+2} \mid 2 \leq k \leq n-4\right\} \cup\left\{\alpha_{k, k+1, k+2} \mid 2 \leq k \leq n-3\right\} \cup \\
& \left\{\alpha_{1,2 k+1}^{e} \mid 2 \leq k \leq \frac{n}{2}-1\right\} \cup\left\{\alpha_{2 k, n} \mid 1 \leq k \leq \frac{n-4}{2}\right\} \cup\left\{\beta_{k, m} \mid 2 \leq k, m \leq n \text { and } k+3 m \leq n-1\right\} .
\end{aligned}
$$

From now on, our main aim is to prove that $G_{n}$ is a generating set for $\mathcal{T F}_{n}$ of minimal size.

The following lemma shows that all the transformations above defined belong to the subsemigroup $\left\langle G_{n}\right\rangle$ of $\mathcal{T F}_{n}$ generated by $G_{n}$. Frequently, we will use it without reference. 
Lemma 3.2. We have:

(i) $\left\{\alpha_{k, k+1, k+2} \mid 1 \leq k \leq n-2\right\} \subseteq\left\langle G_{n}\right\rangle$;

(ii) $\left\{\alpha_{1,2 k+1} \mid 2 \leq k \leq\left\lfloor\frac{n-1}{2}\right\rfloor\right\} \subseteq\left\langle G_{n}\right\rangle$;

(iii) $\left\{\alpha_{k, k+2} \mid 2 \leq k \leq n-4\right\} \subseteq\left\langle G_{n}\right\rangle$;

(iv) $\left\{\beta_{k, m} \mid 2 \leq k, m \leq n\right.$ and $\left.k+3 m \leq n-1\right\} \subseteq\left\langle G_{n}\right\rangle$;

(v) $\alpha_{2 k, n}=\left(\begin{array}{ccccccc}1 & \ldots & 2 k & \overline{2 k+1, n} & \ldots & \overline{\frac{n-1}{2}+k, \frac{n+3}{2}+k} & \frac{n+1}{2}+k \\ 1 & \ldots & 2 k & 2 k+1 & \ldots & \frac{n-1}{2}+k & \frac{n+1}{2}+k\end{array}\right) \in\left\langle G_{n}\right\rangle$, for $n$ odd and $1 \leq k \leq \frac{n-5}{2}$;

(vi) $\alpha_{n-2, n} \in\left\langle G_{n}\right\rangle$.

Proof. (i) For $n$ odd and $\frac{n-1}{2}<k \leq n-2$, we have $\alpha_{k, k+1, k+2}=\gamma_{n} \alpha_{n-k-1, n-k, n-k+1} \gamma_{n} \alpha_{1,2,3}$. On the other hand, for $n$ even, we have $\alpha_{1,2,3}=\alpha_{1,2}^{e} \alpha_{n-1, n}$ and $\alpha_{n-2, n-1, n}=\alpha_{n-1, n} \alpha_{1,2}^{e} \alpha_{1,2,3}$.

(ii) For $n$ even and $2 \leq k \leq \frac{n-2}{2}$, we have $\alpha_{1,2 k+1}=\alpha_{1,2 k+1}^{e} \alpha_{1,2}^{e} \alpha_{1,2,3} \alpha_{1,2}^{e}$.

(iii) For $n$ odd and $\frac{n-1}{2} \leq k \leq n-4$, we have $\alpha_{k, k+2}=\gamma_{n} \alpha_{n-k-2 . n-k} \gamma_{n} \alpha_{1,2,3}$.

(iv) Let $n$ be an odd number and let $k, m \in \bar{n}$ be such that $k+3 m \leq n-1$ and $2 k+3 m>n+1$. Then $2(n-(k+3 m)+1) \leq n+1$ and we have $\beta_{k, m}=\gamma_{n} \beta_{n-(k+3 m)+1, m} \gamma_{n}\left(\alpha_{1,2,3}\right)^{m}$.

(v) For $1 \leq k \leq \frac{n-5}{2}$, we have $\alpha_{2 k, n}=\gamma_{n} \alpha_{1,2(k+1)+1} \gamma_{n}$.

(vi) Finally, we have $\alpha_{n-2, n}=\gamma_{n} \alpha_{1,3} \gamma_{n}$, whenever $n$ is odd.

In order to prove that the set $G_{n}$ generates $\mathcal{T F}_{n}$, our first step is to show that, for any transformation in $\mathcal{T F}_{n}$, there exists a transformation in $\left\langle G_{n}\right\rangle$ with the same kernel. For any set $A \subseteq \bar{n}$, define

$$
\operatorname{Rel}(A)=\{x \in \bar{n} \backslash A \mid x \text { and } a \text { are comparable, for some } a \in A\} .
$$

Lemma 3.3. For any $\alpha \in \mathcal{T F}_{n}$ there exists $\alpha^{\prime} \in\left\langle G_{n}\right\rangle$ such that $\operatorname{Ker} \alpha^{\prime}=\operatorname{Ker} \alpha$.

Proof. Let $\alpha \in \mathcal{T F}_{n}$. We make the proof by induction on the rank of $\alpha$.

If $\operatorname{rank} \alpha=n$ then $\operatorname{Ker} \alpha=\operatorname{Ker}_{n}$ and we have $\operatorname{id}_{n} \in G_{n}$, for $n$ even, and $\operatorname{id}_{n}=\gamma_{n}^{2} \in\left\langle G_{n}\right\rangle$, for $n$ odd.

Assume that $\operatorname{rank} \alpha=n-1$. Then, there exists $i \in \operatorname{Im} \alpha$ such that $\left|i \alpha^{-1}\right|=2$ and $\left|j \alpha^{-1}\right|=1$, for all $j \in \operatorname{Im} \alpha \backslash\{i\}$. This implies $\left|\operatorname{Rel}\left(i \alpha^{-1}\right)\right| \leq 2$, i.e. $i \alpha^{-1}=\{1,2\}$ or $i \alpha^{-1}=\{1,3\}$ or $i \alpha^{-1}=\{n-2, n\}$ or $i \alpha^{-1}=\{n-1, n\}$. By noticing that, for an odd $n$, we have $\alpha_{n-1, n}=\gamma_{n} \alpha_{1,2}$ and $\alpha_{n-2, n}=\gamma_{n} \alpha_{1,3} \gamma_{n}$, it follows that there exists $\alpha^{\prime} \in\left\langle G_{n}\right\rangle$ such that $\operatorname{Ker} \alpha^{\prime}=\operatorname{Ker} \alpha$.

Admit now that $\operatorname{rank} \alpha=n-2$. Then, for some $i \in \operatorname{Im} \alpha$, we have $2 \leq\left|i \alpha^{-1}\right| \leq 3$.

If $\left|i \alpha^{-1}\right|=3$ then there exists $k \in\{1, \ldots, n-2\}$ such that $i \alpha^{-1}=\{k, k+1, k+2\}$ and $\left|j \alpha^{-1}\right|=1$, for all $j \in \operatorname{Im} \alpha \backslash\{i\}$, i.e. $\operatorname{Ker} \alpha=\operatorname{Ker} \alpha_{k, k+1, k+2}$, with $\alpha_{k, k+1, k+2} \in\left\langle G_{n}\right\rangle$.

Now, suppose that $\left|i \alpha^{-1}\right|=2$. Then $\left|j \alpha^{-1}\right|=2$, for some $j \in \operatorname{Im} \alpha \backslash\{i\}$.

Admit that $\left|\operatorname{Rel}\left(i \alpha^{-1}\right)\right| \leq 2$. Then $i \alpha^{-1}=\{1,2\}$ or $i \alpha^{-1}=\{1,3\}$ or $i \alpha^{-1}=\{n-2, n\}$ or $i \alpha^{-1}=\{n-1, n\}$. Since rank $\alpha=n-2$, we conclude that $\left|\operatorname{Rel}\left(j \alpha^{-1}\right)\right| \leq 2$ or $i \alpha^{-1} \subseteq \operatorname{Rel}\left(j \alpha^{-1}\right)$. So, we have $j \alpha^{-1}=\{n-2, n\}$ or $j \alpha^{-1}=\{n-1, n\}$, if $i \alpha^{-1}=\{1,2\}$ or $i \alpha^{-1}=\{1,3\}$, or $j \alpha^{-1}=\{2,4\}$, if $i \alpha^{-1}=\{1,3\}$, or $j \alpha^{-1}=\{n-3, n-1\}$, if $i \alpha^{-1}=\{n-2, n\}$. Hence, we get $\operatorname{Ker} \alpha^{\prime}=\operatorname{Ker} \alpha$, with $\alpha^{\prime}=\alpha_{1,2} \alpha_{n-1, n}$ (and $\alpha^{\prime}=\left(\alpha_{1,2} \gamma_{n}\right)^{2}$, whenever $n$ is odd) or $\alpha^{\prime}=\alpha_{1,2} \alpha_{n-2, n}$ or $\alpha^{\prime}=\alpha_{1,3} \alpha_{n-1, n}$ (and $\alpha^{\prime}=\alpha_{1,3} \gamma_{n} \alpha_{1,2} \gamma_{n}$, whenever $n$ is odd) or $\alpha^{\prime}=\alpha_{1,3} \alpha_{n-2, n}$ or $\alpha^{\prime}=\alpha_{1,3} \alpha_{1,5}$ or $\alpha^{\prime}=\alpha_{n-2, n} \alpha_{n-4, n}$. Observe $\alpha_{n-4, n}=\gamma_{n} \alpha_{1,5} \gamma_{n} \in\left\langle G_{n}\right\rangle$, whenever $n$ is odd (since $\alpha_{1,5} \in\left\langle G_{n}\right\rangle$ by Lemma 3.2), and $\alpha_{1,2}=\alpha_{1,2}^{e} \alpha_{1,2}^{e}$, whenever $n$ is even. Since all the other transformations used belong to $\left\langle G_{n}\right\rangle$, we have $\alpha^{\prime} \in\left\langle G_{n}\right\rangle$. Dually, in the case $\left|\operatorname{Rel}\left(j \alpha^{-1}\right)\right| \leq 2$, we can show that there exists $\alpha^{\prime} \in\left\langle G_{n}\right\rangle$, with $\operatorname{Ker} \alpha^{\prime}=\operatorname{Ker} \alpha$.

Notice that the case $\left|\operatorname{Rel}\left(i \alpha^{-1}\right)\right| \geq 4$ or $\left|\operatorname{Rel}\left(j \alpha^{-1}\right)\right| \geq 4$ is not possible since rank $\alpha=n-2$. So, it remains the case $\left|\operatorname{Rel}\left(i \alpha^{-1}\right)\right|=\left|\operatorname{Rel}\left(j \alpha^{-1}\right)\right|=3$. This provides $i \alpha^{-1}=\{1, k\}$, for some $k \in 2 \mathbb{N}+3$, or $i \alpha^{-1}=\{n-k, n\}$, 
for some $k \in 2 \mathbb{N}+2$, or $i \alpha^{-1}=\{k, k+2\}$ for some $k \in\{2, \ldots, n-3\}$. Then there are two elements in $\operatorname{Rel}\left(j \alpha^{-1}\right)$ with the same image, which is $i$ since $\operatorname{rank} \alpha=n-2$. This shows that $i \alpha^{-1} \subseteq \operatorname{Rel}\left(j \alpha^{-1}\right)$. By the same argumentation, we obtain $j \alpha^{-1} \subseteq \operatorname{Rel}\left(i \alpha^{-1}\right)$.

Suppose that $i \alpha^{-1}=\{1, k\}$, for some $k \in 2 \mathbb{N}+3$. Assume that $k \geq 7$. Then $j \alpha^{-1} \subseteq \operatorname{Rel}\left(i \alpha^{-1}\right)=\{2, k-1, k+$ $1\}$ and $i \alpha^{-1} \subseteq \operatorname{Rel}\left(j \alpha^{-1}\right)$ implies $\left|\operatorname{Rel}\left(j \alpha^{-1}\right)\right|=4$, a contradiction. Hence, we have $i \alpha^{-1}=\{1,5\}$. Then, once again $i \alpha^{-1} \subseteq \operatorname{Rel}\left(j \alpha^{-1}\right)$ and $\left|\operatorname{Rel}\left(j \alpha^{-1}\right)\right|=3$ implies $j \alpha^{-1}=\{2,4\}$. Thus Ker $\alpha=\operatorname{Ker} \alpha_{1,5}$ and $\alpha_{1,5} \in\left\langle G_{n}\right\rangle$. Dually, we can show the existence of $\alpha^{\prime} \in\left\langle G_{n}\right\rangle$ with $\operatorname{Ker} \alpha^{\prime}=\operatorname{Ker} \alpha$, if $i \alpha^{-1}=\{n-k, n\}$, for some $k \in 2 \mathbb{N}+2$. Similarly, we obtain $\alpha^{\prime} \in\left\langle G_{n}\right\rangle$ with $\operatorname{Ker} \alpha^{\prime}=\operatorname{Ker} \alpha$, if $j \alpha^{-1}=\{1, k\}$, for some $k \in 2 \mathbb{N}+3$, or $j \alpha^{-1}=\{n-k, n\}$, for some $k \in 2 \mathbb{N}+2$.

Finally, we consider the case $i \alpha^{-1}=\{k, k+2\}$ and $j \alpha^{-1}=\{\ell, \ell+2\}$, for some $k, \ell \in\{2, \ldots, n-3\}$. Notice that $\{k, k+2\}=i \alpha^{-1} \subseteq \operatorname{Rel}\left(j \alpha^{-1}\right)=\{\ell-1, \ell+1, \ell+3\}$ and so $k=\ell-1$ or $k=\ell+1$. Therefore, we have $\operatorname{Ker} \alpha=\operatorname{Ker} \alpha_{m, m+2}$, with $m=k$, if $k=\ell-1$, or $m=\ell$, if $k=\ell+1$. Hence, Ker $\alpha=\operatorname{Ker} \alpha_{m, m+2}$ and $\alpha_{m, m+2} \in\left\langle G_{n}\right\rangle$.

Next, we suppose that $p=\operatorname{rank} \alpha<n-2$ and assume that for all $\beta \in \mathcal{T F}_{n}$ with $\operatorname{rank} \beta>p$, there exists $\beta^{\prime} \in\left\langle G_{n}\right\rangle$ such that $\operatorname{Ker} \beta^{\prime}=\operatorname{Ker} \beta$. Further, there exist a unique $m \in \bar{n}$, a sequence $a_{1}, \ldots, a_{m} \in \operatorname{Im} \alpha$ and a partition $A_{1}<\cdots<A_{m}$ of $\bar{n}$ with $\left|a_{i}-a_{i+1}\right|=1$, for $1 \leq i<m$, and $A_{i} \alpha=\left\{a_{i}\right\}$, for $1 \leq i \leq m$. Notice that the elements in the sequence $a_{1}, \ldots, a_{m}$ have not to be pairwise distinct and $\operatorname{Im} \alpha=\left\{a_{1}, \ldots, a_{m}\right\}$. Put $\chi(\alpha)=m$. Observe that this construction can be applied to any element of $\mathcal{T F}_{n}$ and so we have a well defined mapping $\chi: \mathcal{T F}_{n} \rightarrow \bar{n}$.

Let

$$
a_{0}= \begin{cases}0 & \text { if } a_{1} \text { is odd } \\ 1 & \text { if } a_{1} \text { is even }\end{cases}
$$

and define

$$
\beta=\left(\begin{array}{cccc}
A_{1} & A_{2} & \cdots & A_{m} \\
1+a_{0} & 2+a_{0} & \cdots & m+a_{0}
\end{array}\right) .
$$

It is clear that $\beta \in \mathcal{T F}_{n}$.

First, consider the case $m=p$ (i.e. $\operatorname{Ker} \alpha=\operatorname{Ker} \beta$ ). Take $i \in\{1, \ldots, p\}$ such that $\left|A_{i}\right| \geq 3$ and $A_{i}=$ $\{k, k+1, \ldots, k+s\}$, with $k \in\{1, \ldots, n-2\}$ and $s \in\{2, \ldots, n-k\}$. Define

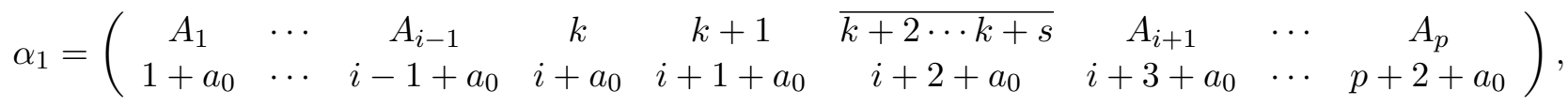

for $i>1$, and

$$
\alpha_{1}=\left(\begin{array}{cccccc}
\overline{1 \cdots k-2+s} & k-1+s & k+s & A_{2} & \cdots & A_{p} \\
1+a_{0} & 2+a_{0} & 3+a_{0} & 4+a_{0} & \cdots & p+2+a_{0}
\end{array}\right),
$$

if $i=1$. Since $p<n-2$, we have $p+2+a_{0} \in \bar{n}$. By using Theorem 2.1, we can verify that $\alpha_{1} \in \mathcal{T F}_{n}$. Since rank $\alpha_{1}>p$, there is $\alpha_{1}^{*} \in\left\langle G_{n}\right\rangle$ with $\operatorname{Ker} \alpha_{1}^{*}=\operatorname{Ker} \alpha_{1}$. Suppose that $\operatorname{Im} \alpha_{1}^{*}=\left\{a_{1}^{*}, \ldots, a_{p+2}^{*}\right\}$ such that $a_{j}^{*}\left(\alpha_{1}^{*}\right)^{-1}=\left(j+a_{0}\right) \alpha_{1}^{-1}$ for $j \in\{1, \ldots, p+2\}$. Let

$$
\alpha_{2}=\left\{\begin{array}{lll}
\alpha_{a_{i}^{*}, a_{i+1}^{*}, a_{i+2}^{*}} & \text { if } & a_{i}^{*}<a_{i+1}^{*} \\
\alpha_{a_{i+2}^{*}, a_{i+1}^{*}, a_{i}^{*}} & \text { if } & a_{i+1}^{*}<a_{i}^{*} .
\end{array}\right.
$$

It is a routine matter to verify that $\operatorname{Ker} \alpha_{1} \alpha_{2}=\operatorname{Ker} \beta$ and so there exists $\alpha^{\prime} \in\left\langle G_{n}\right\rangle$ such that $\operatorname{Ker} \alpha^{\prime}=\operatorname{Ker} \beta=$ $\operatorname{Ker} \alpha$.

Now, admit that $m>p$. Then, there exist $i \in\{1, \ldots, m-1\}$ and $s \in\{i, \ldots, m-i\}$ such that the elements of $\left\{a_{i}, \ldots, a_{i+s}\right\}$ are pairwise distinct, $a_{i+2 s}=a_{i}$ and one of the following five conditions is satisfied:

(a) $i+a_{0}=1$;

(b) $i+a_{0} \geq 2, i+2 s=m$ and $a_{0}+i+2 s=n$; 
(c) $i+a_{0} \geq 2, i+2 s=m, a_{0}+i+2 s<n$ and $n-m<i$;

(d) $i+a_{0} \geq 2, i+2 s=m, a_{0}+i+2 s<n$ and $n-m \geq i$;

(e) $a_{i+3 s}=a_{i+s}$ and $i+3 s<n$.

We will define in each of these five cases transformations $\rho_{1}$ and $\omega_{1}$. Let $\rho_{1}=\alpha_{1,2 s+1}$, in the case (a); let $\rho_{1}=\alpha_{2\left\lfloor\frac{n-2 s}{2}\right\rfloor, n}$, in the case (b); let $\rho_{1}=\alpha_{2\left\lfloor\frac{2(i+s)-n}{2}\right\rfloor, n}$, in the case (c), where $2(i+s)-n=i+m-n>i-i=0$; let $\rho_{1}^{*}$ be defined by

$$
x \rho_{1}^{*}= \begin{cases}2\left(i+s+a_{0}\right)-x & \text { if } 1 \leq x \leq i+s+a_{0} \\ x & \text { otherwise }\end{cases}
$$

in the case (d); and let $\rho_{1}=\beta_{i, s}$, in the case (e). It is easy to verify that $\rho_{1} \in\left\langle G_{n}\right\rangle$ in the cases (a), (b), (c) and (e). In the case $(\mathrm{d})$, we observe that $q=\operatorname{rank} \rho_{1}^{*}=n-\left(i+s+a_{0}\right)+1>p$. Then there exists $\rho_{1} \in\left\langle G_{n}\right\rangle$ such that $\operatorname{Ker} \rho_{1}=\operatorname{Ker} \rho_{1}^{*}$. Suppose that $\operatorname{Im} \rho_{1}=\left\{d_{1}, \ldots, d_{q}\right\}$ such that $j\left(\rho_{1}^{*}\right)^{-1}=d_{j-\left(s+i+a_{0}\right)+1} \rho_{1}^{-1}$ for $i+s+a_{0} \leq j \leq n$. Let $\omega_{1}$ be defined by

$$
x \omega_{1}= \begin{cases}a_{1+s} & \text { if } 1 \leq x \leq 1+s \\ a_{x} & \text { if } 1+s<x<m \\ a_{m} & \text { otherwise }\end{cases}
$$

in the case (a); let $\omega_{1}$ be defined by

$$
x \omega_{1}= \begin{cases}a_{x-a_{0}} & \text { if } 1+a_{0} \leq x<i+s+a_{0} \\ a_{i+s} & \text { if } i+s+a_{0} \leq x \leq n \\ a_{1} & \text { otherwise }\end{cases}
$$

in the cases (b) and (c). Since $\ell$ and $a_{\ell-a_{0}}$ have the same parity for all $1+a_{0} \leq \ell \leq m+a_{0}$, we conclude that $\omega_{1} \in \mathcal{T F}_{n}$. Let $\omega_{1}$ be defined by

$$
x \omega_{1}= \begin{cases}a_{i+s} & \text { if } 1 \leq x \leq d_{1}<d_{2} \text { or } d_{2}<d_{1} \leq x \leq n \\ a_{i+s-\ell+1} & \text { if } x=d_{\ell} \text { and } 1 \leq \ell \leq i+s \\ a_{1} & \text { otherwise }\end{cases}
$$

in the case $(\mathrm{d})$. Let $l \in\{1, \ldots, i+s\}$. Then there exists $j \in\left\{i+s+a_{0}, \ldots, n\right\}$ such that $\ell=j-\left(i+a+a_{0}\right)-1$. From $j\left(\rho_{1}^{*}\right)^{-1}=d_{\ell} \rho_{1}^{-1}, d_{\ell} \omega_{1}=a_{i+s-\ell+1}$ and the fact that $j$ and $a_{j+a_{0}}$ have the same parity, we conclude that $d_{\ell}$ and $d_{\ell} \omega_{1}$ have the same parity. This shows that $\omega_{1} \in \mathcal{T} \mathcal{F}_{n}$. Moreover, $\operatorname{rank} \omega_{1}=\operatorname{rank} \alpha=p$ and $\chi(\alpha)=\chi\left(\omega_{1}\right)+s$. Consider now the case (e) and define $\omega_{1}$ by

$$
x \omega_{1}= \begin{cases}a_{x-a_{0}} & \text { if } 1+a_{0} \leq x \leq i+s+a_{0} \\ a_{2 s+x-a_{0}} & \text { if } i+s+a_{0}+1 \leq x \leq m-2 s+a_{0} \\ a_{m} & \text { if } m-2 s+a_{0}<x \leq n \\ a_{1} & \text { if } x=1 .\end{cases}
$$

It is easy to verify that $\operatorname{rank} \alpha=\operatorname{rank} \omega_{1}$ and $\chi(\alpha)=\chi\left(\omega_{1}\right)+2 s$. Moreover, it is a routine matter to show that $\omega_{1} \in \mathcal{T F}_{n}$ and $\alpha=\beta \rho_{1} \omega_{1}$.

Next, we can focus on $\omega_{1}$ and end up getting a sequence $\rho_{1}, \ldots, \rho_{t} \in\left\langle G_{n}\right\rangle$ (for a suitable $t \in \mathbb{N}$ ) and an element $\omega \in \mathcal{T F}_{n}$ such that $\operatorname{rank} \alpha=\operatorname{rank} \omega, \chi(\omega)=p$ and $\alpha=\beta \rho_{1} \cdots \rho_{t} \omega$.

By the case $m=p$, there exists $\omega^{\prime} \in\left\langle G_{n}\right\rangle$ such that $\operatorname{Ker} \omega^{\prime}=\operatorname{Ker} \omega$, whence $\operatorname{Ker} \beta \rho_{1} \cdots \rho_{t} \omega^{\prime}=\operatorname{Ker} \alpha$.

On the other hand, since $m>p$, there exists $\mu \in\left\langle G_{n}\right\rangle$ such that Ker $\mu=\left\{A_{1}, \ldots, A_{m}\right\}$, say

$$
\mu=\left(\begin{array}{cccc}
A_{1} & A_{2} & \cdots & A_{m} \\
c_{1} & c_{2} & \cdots & c_{m}
\end{array}\right)
$$

by our inductive assumption. Clearly, by Theorem 2.1, either $c_{1}>\cdots>c_{m}$ or $c_{1}<\cdots<c_{m}$. If $c_{1}>\cdots>c_{m}$ then we take $\varepsilon_{1}=\alpha_{1,2}^{e}$, if $n$ is even, and we take $\varepsilon_{1}=\gamma_{n}$, if $n$ is odd. Since $\varepsilon_{1} \in G_{n}$, whence $\mu \varepsilon_{1} \in\left\langle G_{n}\right\rangle$, we 
can assume that $c_{1}<\cdots<c_{m}$. If $1+a_{0}<c_{1}$ then there exists $s \in \bar{n}$ such that $1+a_{0}=c_{1}-2 s$. It follows that $\beta=\mu\left(\alpha_{1,2,3}\right)^{s}$ and so $\beta \in\left\langle G_{n}\right\rangle$.

Altogether, we have shown that $\beta \rho_{1} \cdots \rho_{t} w^{\prime} \in\left\langle G_{n}\right\rangle$ and $\operatorname{Ker} \beta \rho_{1} \cdots \rho_{t} w^{\prime}=\operatorname{Ker} \alpha$, as required.

Now, we are able to prove that $G_{n}$ is a generating set for $\mathcal{T F}_{n}$.

Proposition 3.4. We have $\left\langle G_{n}\right\rangle=\mathcal{T F}_{n}$.

Proof. Let $\alpha \in \mathcal{T F}_{n}$.

Admit that $\operatorname{rank} \alpha=n$. If $n$ is even then $\alpha=\operatorname{id}_{n} \in G_{n}$. If $n$ is odd then $\alpha=\operatorname{id}_{n}$ or $\alpha=\gamma_{n} \in G_{n}$, with $\gamma_{n} \gamma_{n}=\mathrm{id}_{n}$. Thus $\alpha \in\left\langle G_{n}\right\rangle$.

Suppose now that $2 \leq m=\operatorname{rank} \alpha<n$. By Lemma 3.3, there exists $\alpha^{\prime} \in\left\langle G_{n}\right\rangle$ such that $\operatorname{Ker} \alpha=\operatorname{Ker} \alpha^{\prime}$. Take

$$
\operatorname{Im} \alpha=\left\{a_{1}, \ldots, a_{m}\right\} \quad \text { and } \quad \operatorname{Im} \alpha^{\prime}=\left\{a_{1}^{\prime}, \ldots, a_{m}^{\prime}\right\}
$$

with $a_{1}<a_{2}<\cdots<a_{m}$ and $a_{1}^{\prime}<a_{2}^{\prime}<\cdots<a_{m}^{\prime}$, and define $A_{i}=a_{i} \alpha^{-1}$, for $1 \leq i \leq m$. Observe that $A_{i}=a_{i}^{\prime} \alpha^{\prime-1}$, for $1 \leq i \leq m$, or $A_{i}=a_{m-i+1}^{\prime} \alpha^{\prime-1}$, for $1 \leq i \leq m$.

Let $m=n-1$. Then $n \notin \operatorname{Im} \alpha$ or $1 \notin \operatorname{Im} \alpha$ as well as $n \notin \operatorname{Im} \alpha^{\prime}$ or $1 \notin \operatorname{Im} \alpha^{\prime}$.

If $A_{i}=a_{i}^{\prime} \alpha^{\prime-1}$, for $1 \leq i \leq n-1$ then $a_{1}=a_{1}^{\prime}$, since $a_{1}$ and $a_{1}^{\prime}$ have the same parity, by Lemma 3.1. Hence, $a_{i}=a_{i}^{\prime}$, for $1 \leq i \leq n-1$, and so $\alpha=\alpha^{\prime}$.

Next consider the case $A_{i}=a_{m-i+1}^{\prime} \alpha^{\prime-1}$, for $1 \leq i \leq n-1$. Let

$$
k= \begin{cases}0 & \text { if } a_{1}=1 \\ 1 & \text { if } a_{1}=2\end{cases}
$$

Then, $a_{i}=i+k$ and

$$
a_{m-i+1}^{\prime}= \begin{cases}n-k-i+1 & \text { if } n \text { is odd } \\ n+k-i & \text { if } n \text { is even }\end{cases}
$$

for $i=1, \ldots, n-1$. If $n$ is odd, then we have

$$
a_{i}\left(\alpha^{\prime} \gamma_{n}\right)^{-1}=(i+k) \gamma_{n}^{-1} \alpha^{\prime-1}=(n-(i+k)+1) \alpha^{\prime-1}=a_{m-i+1}^{\prime} \alpha^{\prime-1}=A_{i}=a_{i} \alpha^{-1},
$$

for $1 \leq i \leq n-1$. Since $\operatorname{Ker} \alpha=\operatorname{Ker} \alpha^{\prime}=\operatorname{Ker} \alpha / \gamma_{n}$, this shows that $\alpha=\alpha^{\prime} \gamma_{n} \in\left\langle G_{n}\right\rangle$. If $n$ is even then put $\rho_{0}=\alpha_{n-1, n} \in\left\langle G_{n}\right\rangle$ and $\rho_{1}=\alpha_{1,2}^{e} \in\left\langle G_{n}\right\rangle$. Observe that $\rho_{k}$ restricted to $\operatorname{Im} \alpha^{\prime}$ is an injection. Hence, we have $\operatorname{Ker} \alpha=\operatorname{Ker} \alpha^{\prime}=\operatorname{Ker} \alpha^{\prime} \rho_{k}$ and

$$
a_{i}\left(\alpha^{\prime} \rho_{k}\right)^{-1}=(i+k) \rho_{k}^{-1} \alpha^{\prime-1}=(n-i+k) \alpha^{\prime-1}=A_{i}=a_{i} \alpha^{-1}
$$

for $1 \leq i \leq n-1$. Thus $\alpha=\alpha^{\prime} \rho_{k} \in\left\langle G_{n}\right\rangle$.

Admit now that $2 \leq m \leq n-2$ and suppose that $\beta \in\left\langle G_{n}\right\rangle$, for all $\beta \in \mathcal{T F}_{n}$ such that $\operatorname{rank} \beta>m$.

Suppose that $A_{i}=a_{m-i+1}^{\prime} \alpha^{\prime-1}$, for $1 \leq i \leq m$. Take

$$
\rho= \begin{cases}\gamma_{n} & \text { if } n \text { is odd } \\ \alpha_{1,2}^{e} & \text { if } n \text { is even and } 1 \notin \operatorname{Im} \alpha \\ \alpha_{n-1, n} & \text { if } n \text { is even and } 1 \in \operatorname{Im} \alpha .\end{cases}
$$

Then, we have $\operatorname{Ker} \alpha=\operatorname{Ker} \alpha^{\prime}=\operatorname{Ker} \alpha^{\prime} \rho$ and

$$
A_{i}=a_{m-i+1}^{\prime} \alpha^{\prime-1}=\left(a_{m-i+1}^{\prime} \rho\right) \rho^{-1} \alpha^{-1}=\left(a_{m-i+1}^{\prime} \rho\right)\left(\alpha^{\prime} \rho\right)^{-1},
$$

for $1 \leq i \leq m$, with $\alpha^{\prime} \rho \in\left\langle G_{n}\right\rangle$ and $a_{m-i+1}^{\prime} \rho<a_{m-j+1}^{\prime} \rho$, for $1 \leq i<j \leq m$. Thus, we can assume that $A_{i}=a_{i}^{\prime} \alpha^{\prime-1}$, for $1 \leq i \leq m$.

If $a_{1}=a_{1}^{\prime}=1$ then we immediately obtain that $a_{i}=a_{i}^{\prime}$, for $1 \leq i \leq m$, i.e. $\alpha=\alpha^{\prime} \in\left\langle G_{n}\right\rangle$. 
Consider $a_{1}=1, a_{1}^{\prime}>1$ and $a_{m}^{\prime} \neq n$. This implies $a_{m}^{\prime}, a_{m}<n$ and so we put

$$
\beta_{0}=\left(\begin{array}{ccccc}
\overline{1 \cdots a_{1}^{\prime}} & a_{2}^{\prime} & \cdots & a_{m}^{\prime} & \overline{a_{m}^{\prime}+1 \cdots n} \\
a_{1} & a_{2} & \cdots & a_{m} & a_{m}+1
\end{array}\right) .
$$

It is easy to show that $\beta_{0} \in \mathcal{T F}_{n}$, with $\operatorname{rank} \beta_{0}=\operatorname{rank} \alpha+1$, whence $\beta_{0} \in\left\langle G_{n}\right\rangle$. For $1 \leq i \leq m$, we have

$$
a_{i}\left(\alpha^{\prime} \beta_{0}\right)^{-1}=a_{i} \beta_{0}^{-1} \alpha^{\prime-1}=a_{i}^{\prime} \alpha^{\prime-1}=A_{i}=a_{i} \alpha^{-1},
$$

as $a_{i}$ is the unique element in $\operatorname{Im} \alpha^{\prime} \cap a_{i} \beta_{0}^{-1}$. Since the restriction of $\beta_{0}$ to $\operatorname{Im} \alpha^{\prime}$ is injective, we also have $\operatorname{Ker} \alpha=\operatorname{Ker} \alpha^{\prime}=\operatorname{Ker} \alpha^{\prime} \beta_{0}$. Thus $\alpha=\alpha^{\prime} \beta_{0} \in\left\langle G_{n}\right\rangle$.

Next, consider $a_{1}=1, a_{1}^{\prime}>1$ and $a_{m}^{\prime}=n$. Then $a_{1}^{\prime} \geq 3$, since $a_{1}$ and $a_{1}^{\prime}$ have the same parity. Further, we have $a_{i}=i$, for $1 \leq i \leq m$. So, we obtain

$$
\beta_{1}=\left(\begin{array}{ccccc}
\overline{1,3} & \overline{2,4} & 5 & \cdots & n \\
1 & 2 & 3 & \cdots & n-2
\end{array}\right)= \begin{cases}\alpha_{1,3} \alpha_{1,5}^{e} \in\left\langle G_{n}\right\rangle & \text { if } n \text { is even } \\
\alpha_{1,3} \alpha_{1,5} \alpha_{1,2,3} \in\left\langle G_{n}\right\rangle & \text { if } n \text { is odd. }\end{cases}
$$

Moreover, let

$$
\beta_{2}=\left(\begin{array}{cccccc}
1 & \overline{2 \cdots a_{1}^{\prime}-1} & a_{1}^{\prime} & \cdots & a_{m-1}^{\prime} & \overline{a_{m}^{\prime} \cdots n} \\
1 & 2 & 3 & \cdots & m+1 & m+2
\end{array}\right) .
$$

It is easy to verify that $\beta_{2} \in \mathcal{T} \mathcal{F}_{n}$, with $\operatorname{rank} \beta_{2}=\operatorname{rank} \alpha+2>m$, whence $\beta_{2} \in\left\langle G_{n}\right\rangle$. Hence

$$
\begin{gathered}
a_{1}\left(\alpha^{\prime} \beta_{2} \beta_{1}\right)^{-1}=a_{1} \beta_{1}^{-1} \beta_{2}^{-1} \alpha^{\prime-1}=1 \beta_{1}^{-1} \beta_{2}^{-1} \alpha^{\prime-1}=\{1,3\} \beta_{2}^{-1} \alpha^{\prime-1}=\left\{1, a_{1}^{\prime}\right\} \alpha^{\prime-1}=a_{1}^{\prime} \alpha^{\prime-1}=A_{1}=a_{1} \alpha^{-1}, \\
a_{2}\left(\alpha^{\prime} \beta_{2} \beta_{1}\right)^{-1}=2 \beta_{1}^{-1} \beta_{2}^{-1} \alpha^{\prime-1}=\{2,4\} \beta_{2}^{-1} \alpha^{\prime-1}=\left\{2, \ldots, a_{1}^{\prime}-1, a_{2}^{\prime}\right\} \alpha^{\prime-1}=a_{2}^{\prime} \alpha^{\prime-1}=A_{2}=a_{2} \alpha^{-1}
\end{gathered}
$$

and, for $3 \leq i \leq m$,

$$
a_{i}\left(\alpha^{\prime} \beta_{2} \beta_{1}\right)^{-1}=i \beta_{1}^{-1} \beta_{2}^{-1} \alpha^{\prime-1}=(i+2) \beta_{2}^{-1} \alpha^{\prime-1}=a_{i}^{\prime} \alpha^{\prime-1}=A_{i}=a_{i} \alpha^{-1} .
$$

Notice that $\beta_{2}$ restricted to $\operatorname{Im} \alpha^{\prime}$ and $\beta_{1}$ restricted to $\operatorname{Im} \alpha^{\prime} \beta_{2}=\{3, \ldots, m+2\}$ are injective. It follows that $\operatorname{Ker} \alpha=\operatorname{Ker} \alpha^{\prime} \beta_{2} \beta_{1}$ and so $\alpha=\alpha^{\prime} \beta_{2} \beta_{1} \in\left\langle G_{n}\right\rangle$.

Now, consider $a_{1}>1$. Suppose that $a_{1}^{\prime}=1$. Then $a_{m}^{\prime}<n-1$, since rank $\alpha^{\prime} \leq n-2$. Take

$$
\beta_{3}=\left(\begin{array}{ccccc}
1 & 2 & \cdots & n-3 & \overline{n-2, n-1, n} \\
3 & 4 & \cdots & n-1 & n
\end{array}\right) .
$$

If $n$ is even then $\beta_{3}=\alpha_{n-1, n} \alpha_{1,2}^{e}$, whence $\beta_{3} \in\left\langle G_{n}\right\rangle$. On the other hand, if $n$ is odd then $\beta_{3}=\gamma_{n} \alpha_{1,2,3} \gamma_{n} \in\left\langle G_{n}\right\rangle$. Thus, we have $\alpha^{\prime} \beta_{3} \in\left\langle G_{n}\right\rangle$. Clearly, $1 \notin \operatorname{Im} \beta_{3}$ and so $1 \notin \operatorname{Im} \alpha^{\prime} \beta_{3}$. Since $n, n-1 \notin \operatorname{Im} \alpha^{\prime}$, we have that $\beta_{3}$ restricted to $\operatorname{Im} \alpha^{\prime}$ is injective. Hence $\operatorname{Ker} \alpha^{\prime}=\operatorname{Ker} \alpha^{\prime} \beta_{3}$. Therefore, we can assume that $a_{1}^{\prime}>1$. Take

$$
\beta_{4}=\left(\begin{array}{ccccc}
\overline{1 \cdots a_{1}^{\prime}-1} & a_{1}^{\prime} & \cdots & a_{m-1}^{\prime} & \overline{a_{m}^{\prime} \cdots n} \\
a_{1}-1 & a_{1} & \cdots & a_{m-1} & a_{m}
\end{array}\right) .
$$

It is easy to verify that $\beta_{4} \in \mathcal{T F}_{n}$, with $\operatorname{rank} \beta_{4}=\operatorname{rank} \alpha+1>m$, whence $\beta_{4} \in\left\langle G_{n}\right\rangle$. Since $\beta_{4}$ restricted to $\operatorname{Im} \alpha^{\prime}$ is injective, we obtain $\operatorname{Ker} \alpha=\operatorname{Ker} \alpha^{\prime}=\operatorname{Ker} \alpha^{\prime} \beta_{4}$ and, for $i \in\{1, \ldots, m\}$, we have

$$
a_{i}\left(\alpha^{\prime} \beta_{4}\right)^{-1}=a_{i} \beta_{4}^{-1} \alpha^{\prime-1}=a_{i}^{\prime} \alpha^{\prime-1}=A_{i}=a_{i} \alpha^{-1} .
$$

Thus $\alpha=\alpha^{\prime} \beta_{4} \in\left\langle G_{n}\right\rangle$.

Finally, let $m=1$, i.e. there exists $a \in \bar{n}$ such that $i \alpha=a$, for all $i \in \bar{n}$. Without loss of generality, suppose that $a>1$. Clearly, $\beta_{5}=\left(\begin{array}{cc}1 & \overline{2 \cdots n} \\ 1 & 2\end{array}\right) \in\left\langle G_{n}\right\rangle$ and either $\beta_{6}=\left(\begin{array}{cc}\overline{1,2} & \overline{3 \cdots n} \\ a & a-1\end{array}\right) \in\left\langle G_{n}\right\rangle$ (if $a$ is even) or $\beta_{6}=\left(\begin{array}{ll}\overline{1,2} & \overline{3 \cdots n} \\ a-1 & a\end{array}\right) \in\left\langle G_{n}\right\rangle$ (if $a$ is odd). Then $\beta_{5} \beta_{6}$ is the constant mapping with image $\{a\}$, i.e. $\alpha=\beta_{5} \beta_{6} \in\left\langle G_{n}\right\rangle$, as required. 
It remains to show that $G_{n}$ is a generating set for $\mathcal{T F}_{n}$ of minimal size. With this goal in mind, in the next two lemmas, we determine a lower bound for the minimal size of a generating set for $\mathcal{T F}_{n}$ (for $n$ odd as well as for $n$ even) and find it coincides with the cardinality of $G_{n}$ (which gives us an upper bound).

First, we consider an odd $n$.

Lemma 3.5. Let $n$ be an odd number. Then $\operatorname{rank}\left(\mathcal{T F}_{n}\right) \geq \frac{3}{2}(n-1)+\sum_{k=2}^{\frac{n-5}{2}}\left(\left\lfloor\frac{n+1-2 k}{3}\right\rfloor-1\right)=\left|G_{n}\right|$.

Proof. Let $A$ be a generating set of $\mathcal{T F}_{n}$.

Since $\left\{\alpha \in \mathcal{T F}_{n} \mid \operatorname{rank} \alpha=n\right\}=\left\{\gamma_{n}, \mathrm{id}_{n}\right\}$, we have $\gamma_{n} \in A$. Let $A^{(0)}=\left\{\gamma_{n}\right\}$. Then $\left|A^{(0)}\right|=1$.

Let $\alpha \in \mathcal{T F}_{n}$ be such that rank $\alpha \leq n-1$. Then, for some natural number $p$, there exist $\alpha_{1}, \ldots, \alpha_{p} \in A \backslash\left\{\operatorname{id}_{n}\right\}$, with $\alpha_{1} \neq \gamma_{n}$, such that $\alpha=\alpha_{1} \cdots \alpha_{p}$ or $\alpha=\gamma_{n} \alpha_{1} \cdots \alpha_{p}$. Take

$$
\alpha_{1}^{*}= \begin{cases}\alpha_{1} & \text { if } \alpha=\alpha_{1} \cdots \alpha_{p} \\ \gamma_{n} \alpha_{1} & \text { if } \alpha=\gamma_{n} \alpha_{1} \cdots \alpha_{p} .\end{cases}
$$

Clearly, $\operatorname{Ker} \alpha_{1}^{*} \subseteq \operatorname{Ker} \alpha$ and $\operatorname{rank} \alpha_{1}^{*} \leq n-1$.

If $\alpha=\alpha_{1,2}$ then $\operatorname{Ker} \alpha_{1}^{*}=\operatorname{Ker} \alpha_{1,2}$ or $\operatorname{Ker} \alpha_{1}^{*}=\operatorname{Ker} \gamma_{n} \alpha_{1,2}$, i.e. there exists $\rho_{1,2} \in A$ with $\operatorname{Ker} \rho_{1,2}=\operatorname{Ker} \alpha_{1,2}$ or $\operatorname{Ker} \rho_{1,2}=\operatorname{Ker} \gamma_{n} \alpha_{1,2}$ (namely $\rho_{1,2}=\alpha_{1}$ ). Take $A^{(1)}=A^{(0)} \cup\left\{\rho_{1,2}\right\}$. Then $\left|A^{(1)}\right|=\left|A^{(0)}\right|+\left|\left\{\rho_{1,2}\right\}\right|=2$. Analogously, there exists $\rho_{1,3} \in A$ with $\operatorname{Ker} \rho_{1,3}=\operatorname{Ker} \alpha_{1,3}$ or $\operatorname{Ker} \rho_{1,3}=\operatorname{Ker} \gamma_{n} \alpha_{1,3}$. Clearly, $\rho_{1,3} \notin A^{(1)}$ and we take $A^{(2)}=A^{(1)} \cup\left\{\rho_{1,3}\right\}$. Then $\left|A^{(2)}\right|=\left|A^{(1)}\right|+\left|\left\{\rho_{1,3}\right\}\right|=2+1=3$.

Let $\alpha=\alpha_{k, k+2}$, for some $k \in\left\{2, \ldots, \frac{n-3}{2}\right\}$. Then $(k, k+2) \in \operatorname{Ker} \alpha_{1}^{*}$ or $(k+1, k+3) \in \operatorname{Ker} \alpha_{1}^{*}$. From $2 \leq k \leq \frac{n-3}{2}$, it follows that $k+3<n$. Hence, $|\operatorname{Rel}(\{k, k+2\})|=|\operatorname{Rel}(\{k+1, k+3\})|=3$ and there exist $a, b \in \bar{n} \backslash\{k, k+2\}$ or $a, b \in \bar{n} \backslash\{k+1, k+3\}$ such that $(a, b) \in \operatorname{Ker} \alpha_{1}^{*}$. But Ker $\alpha_{1}^{*} \subseteq \operatorname{Ker} \alpha_{k, k+2}$ implies that $(a, b) \in \operatorname{Ker} \alpha_{k, k+2}$. Since $\operatorname{rank} \alpha_{k, k+2}=n-2$, we have $\operatorname{Ker} \alpha_{1}^{*}=\operatorname{Ker} \alpha_{k, k+2}$. Hence, there exists $\rho_{k, k+2} \in A$ with $\operatorname{Ker} \rho_{k, k+2}=\operatorname{Ker} \alpha_{k, k+2}$ or $\operatorname{Ker} \rho_{k, k+2}=\operatorname{Ker} \gamma_{n} \alpha_{k, k+2}$. Moreover, we have $\rho_{k, k+2} \notin A^{(2)}$. On the other hand, assume there exist $2 \leq k<\ell \leq \frac{n-3}{2}$ such that $\operatorname{Ker} \alpha_{k, k+2}=\operatorname{Ker} \gamma_{n} \alpha_{\ell, \ell+2}$. Then $k=n-(\ell+3)+1$ and so $n=k+\ell+3-1<\frac{n-3}{2}+\frac{n-3}{2}+2=n-3+2=n-1$, a contradiction. Hence $\rho_{k, k+2} \neq \rho_{\ell, \ell+2}$, for $2 \leq k<\ell \leq \frac{n-3}{2}$. Take

$$
B^{(3)}=\left\{\rho_{k, k+2} \mid k \in\left\{2, \ldots, \frac{n-3}{2}\right\}\right\}
$$

and $A^{(3)}=A^{(2)} \cup B^{(3)}$. Since $A^{(2)} \cap B^{(3)}=\emptyset$, we obtain $\left|A^{(3)}\right|=\left|A^{(2)}\right|+\left|B^{(3)}\right|=3+\frac{n-5}{2}=\frac{n+1}{2}$.

Let $\alpha=\alpha_{k, k+1, k+2}$, for some $k \in\left\{2, \ldots, \frac{n-1}{2}\right\}$. Then $k+2<n$ and, by Theorem 2.1, there exists no $\beta \in \mathcal{T F}_{n}$ with $\operatorname{rank} \beta=n-1$ such that $\operatorname{Ker} \beta \subseteq \operatorname{Ker} \alpha_{k, k+1, k+2}$. Hence, Ker $\alpha_{1}^{*}=\operatorname{Ker} \alpha_{k, k+1, k+2}$ and so there exists $\rho_{k, k+1, k+2} \in A$ with $\operatorname{Ker} \rho_{k, k+1, k+2}=\operatorname{Ker} \alpha_{k, k+1, k+2}$ or $\operatorname{Ker} \rho_{k, k+1, k+2}=\operatorname{Ker} \gamma_{n} \alpha_{k, k+1, k+2}$. Clearly, $\rho_{k, k+1, k+2} \notin A^{(3)}$.

Let $\alpha=\alpha_{1,2,3}$. If $\operatorname{rank} \alpha_{1}^{*}=n-2$ then $\operatorname{Ker} \alpha_{1}^{*}=\operatorname{Ker} \alpha_{1,2,3}$ or $\operatorname{Ker} \alpha_{1}^{*}=\operatorname{Ker} \gamma_{n} \alpha_{1,2,3}$. Now, admit that $\operatorname{rank} \alpha_{1}^{*}=n-1$. Then there exists $j \in\{2, \ldots, p\}$ such that rank $\alpha_{1}^{*} \alpha_{2} \ldots \alpha_{j-1}=n-1$ and $\operatorname{rank} \alpha_{1}^{*} \alpha_{2} \ldots \alpha_{j}=$ $n-2$. Observe that either $\operatorname{Im} \alpha_{1}^{*} \alpha_{2} \ldots \alpha_{j-1}=\{1, \ldots, n-1\}$, with $\{1,2,3\} \alpha_{1}^{*} \alpha_{2} \ldots \alpha_{j-1}=\{n-2, n-1\}$, or $\operatorname{Im} \alpha_{1}^{*} \alpha_{2} \ldots \alpha_{j-1}=\{2, \ldots, n\}$, with $\{1,2,3\} \alpha_{1}^{*} \alpha_{2} \ldots \alpha_{j-1}=\{2,3\}$. Suppose that $\operatorname{Im} \alpha_{1}^{*} \alpha_{2} \ldots \alpha_{j-1}=\{2, \ldots, n\}$. Then $\{1,2,3\} \alpha_{1}^{*} \alpha_{2} \ldots \alpha_{j-1}=\{2,3\}$ and we conclude that $(2,3) \in \operatorname{Ker} \alpha_{j}$. By Theorem 2.1, this implies that $(1,2) \in \operatorname{Ker} \alpha_{j}$ or $(3,4) \in \operatorname{Ker} \alpha_{j}$. The case $(3,4) \in \operatorname{Ker} \alpha_{j}$ is not possible since otherwise rank $\alpha_{1}^{*} \alpha_{2} \ldots \alpha_{j} \leq n-3$, a contradiction. Thus $(1,2) \in \operatorname{Ker} \alpha_{j}$ and so $\operatorname{Ker} \alpha_{j}=\operatorname{Ker} \alpha_{1,2,3}$. If $\operatorname{Im} \alpha_{1}^{*} \alpha_{2} \ldots \alpha_{j-1}=\{1, \ldots, n-1\}$ then, similarly, we obtain $\operatorname{Ker} \alpha_{j}=\operatorname{Ker} \alpha_{n-2, n-1, n}=\operatorname{Ker} \gamma_{n} \alpha_{1,2,3}$. Therefore, there exists $\rho_{1,2,3} \in A$ with $\operatorname{Ker} \rho_{1,2,3}=$ $\operatorname{Ker} \alpha_{1,2,3}$ or $\operatorname{Ker} \rho_{1,2,3}=\operatorname{Ker} \gamma_{n} \alpha_{1,2,3}$. Clearly, $\rho_{1,2,3} \notin A^{(3)}$. Assume there exist $1 \leq k<\ell \leq \frac{n-1}{2}$ such that $\operatorname{Ker} \alpha_{k, k+1, k+2}=\operatorname{Ker} \gamma_{n} \alpha_{\ell, \ell+1, \ell+2}$. Then $k=n-(\ell+2)+1$ and so $n=\ell+k+1<\frac{n-1}{2}+\frac{n-1}{2}+1=n-1+1=n$, a contradiction. Hence $\rho_{k, k+1, k+2} \neq \rho_{\ell, \ell+1, \ell+2}$, for $1 \leq k<\ell \leq \frac{n-1}{2}$. Take

$$
B^{(4)}=\left\{\rho_{k, k+1, k+2} \mid k \in\left\{1, \ldots, \frac{n-1}{2}\right\}\right\}
$$

and $A^{(4)}=A^{(3)} \cup B^{(4)}$. Since, $A^{(3)} \cap B^{(4)}=\emptyset$, we obtain $\left|A^{(4)}\right|=\left|A^{(3)}\right|+\left|B^{(4)}\right|=\frac{n+1}{2}+\frac{n-1}{2}=n$. 
Let $\alpha=\alpha_{1,2 k+1}$, for some $k \in\left\{2, \ldots, \frac{n-1}{2}\right\}$. Then

$$
\operatorname{Ker} \alpha_{1,2 k+1}=\{(1+i, 2 k+1-i) \mid 0 \leq i \leq k-1\} \cup\{(x, x) \mid x \in \bar{n}\} .
$$

Given $i \in\{1, \ldots, k-2\}$ such that $(1+i, 2 k+1-i) \in \operatorname{Ker} \alpha_{1}^{*}$, we have

$$
\operatorname{Rel}(\{1+i, 2 k+1-i\})=\{1+i-1,2 k+1-i-1,1+i+1,2 k+1-i+1\} .
$$

Since Ker $\alpha_{1}^{*} \subseteq \operatorname{Ker} \alpha_{1,2 k+1}$, we have $(1+(i+1), 2 k+1-(i+1)),(1+(i-1), 2 k+1-(i-1)) \in \operatorname{Ker} \alpha_{1}^{*}$. If $(k, k+2) \in \operatorname{Ker} \alpha_{1}^{*}$ then $\operatorname{Rel}(\{k, k+2\})=\{k-1, k+1, k+3\}$ and so we have $(k-1, k+3) \in \operatorname{Ker} \alpha_{1}^{*}$. Now, assume that $(1+i, 2 k+1-i) \notin \operatorname{Ker} \alpha_{1}^{*}$, for all $i \in\{1, \ldots, k-1\}$. Then $\operatorname{Ker} \alpha_{1}^{*} \subseteq \operatorname{Ker} \alpha_{1,2 k+1} \operatorname{implies}(1,2 k+1) \in \operatorname{Ker} \alpha_{1}^{*}$ and $\operatorname{rank} \alpha_{1}^{*}=n-1$, which is not possible by Theorem 2.1. Therefore, Ker $\alpha_{1}^{*}=\operatorname{Ker} \alpha_{1,2 k+1}$ and so there exists $\rho_{1,2 k+1} \in A$ with $\operatorname{Ker} \rho_{1,2 k+1}=\operatorname{Ker} \alpha_{1,2 k+1}$ or $\operatorname{Ker} \rho_{1,2 k+1}=\operatorname{Ker} \gamma_{n} \alpha_{1,2 k+1}$. Since $(1,2 k+1) \in \operatorname{Ker} \rho_{1,2 k+1}$ or $(n, n-2 k) \in \operatorname{Ker} \rho_{1,2 k+1}$, we have $\rho_{1,2 k+1} \notin A^{(4)}$. For $k, l \in\left\{2, \ldots, \frac{n-1}{2}\right\}$, we have $(1,2 k+1) \in \operatorname{Ker} \alpha_{1,2 k+1}$ and $(1,2 k+1) \notin \operatorname{Ker} \gamma_{n} \alpha_{1,2 \ell+1}$. Hence $\rho_{1,2 k+1} \neq \rho_{1,2 \ell+1}$, for $2 \leq k<\ell \leq \frac{n-1}{2}$. Take

$$
B^{(5)}=\left\{\rho_{1,2 k+1} \mid k \in\left\{2, \ldots, \frac{n-1}{2}\right\}\right\}
$$

and $A^{(5)}=A^{(4)} \cup B^{(5)}$. Since $A^{(4)} \cap B^{(5)}=\emptyset$, we obtain $\left|A^{(5)}\right|=\left|A^{(4)}\right|+\left|B^{(5)}\right|=n+\frac{n-3}{2}=\frac{3 n-3}{2}=\frac{3}{2}(n-1)$.

Finally, let $\alpha=\beta_{k, m}$, for some $k, m \in\left\{2, \ldots, \frac{n-1}{2}\right\}$ such that $2 k+3 m \leq n+1$. It is easy to verify that $\{k+i, k+2 m-i, k+2 m+i\}$, for $0 \leq i \leq m$, are all the non-singleton Ker $\beta_{k, m}$-classes. If $i \in\{1, \ldots, m-1\}$ is such that $(k+i) \alpha_{1}^{*}=(k+2 m-i) \alpha_{1}^{*}=(k+2 m+i) \alpha_{1}^{*}$ then

$\operatorname{Rel}(\{k+i, k+2 m-i, k+2 m+i\})=\{k+i-1, k+2 m-i-1, k+2 m+i-1, k+i+1, k+2 m-i+1, k+2 m+i+1\}$

implies

$$
(k+(i-1)) \alpha_{1}^{*}=(k+2 m-(i-1)) \alpha_{1}^{*}=(k+2 m+(i-1)) \alpha_{1}^{*}
$$

and

$$
(k+(i+1)) \alpha_{1}^{*}=(k+2 m-(i+1)) \alpha_{1}^{*}=(k+2 m+(i+1)) \alpha_{1}^{*},
$$

since $\operatorname{Ker} \alpha_{1}^{*} \subseteq \operatorname{Ker} \beta_{k, m}$. If $(k, k+2 m) \in \operatorname{Ker} \alpha_{1}^{*}$ then, similarly, we have

$$
(k+1) \alpha_{1}^{*}=(k+2 m-1) \alpha_{1}^{*}=(k+2 m+1) \alpha_{1}^{*} .
$$

Moreover, we obtain

$$
(k+m-1) \alpha_{1}^{*}=(k+2 m-(m-1)) \alpha_{1}^{*}=(k+2 m+(m-1)) \alpha_{1}^{*},
$$

whenever $(k+m, k+3 m) \in \operatorname{Ker} \alpha_{1}^{*}$. Therefore $\operatorname{Ker} \alpha_{1}^{*}=\operatorname{Ker} \beta_{k, m}$ and so there exists $\delta_{k, m} \in A$ with $\operatorname{Ker} \delta_{k, m}=$ $\operatorname{Ker} \beta_{k, m}$ or $\operatorname{Ker} \delta_{k, m}=\operatorname{Ker} \gamma_{n} \beta_{k, m}$. Moreover, it is easy to verify that $\delta_{k, m} \notin A^{(5)}$. Take

$$
B^{(6)}=\left\{\delta_{k, m} \mid k, m \in\left\{2, \ldots, \frac{n-1}{2}\right\} \text { and } 2 k+3 m \leq n+1\right\} .
$$

Assume there exist $k, m, p, q \in\left\{2, \ldots, \frac{n-1}{2}\right\}$ such that $\beta_{k, m}=\gamma_{n} \beta_{p, q}$, with $2 k+3 m, 2 p+3 q \leq n+1$ and $k \neq p$ or $m \neq q$. Then $k=n-(p+3 q)+1$. If $k<p$ then $n=k+p+3 q-1<2 p+3 q-1 \leq n+1-1=n$, a contradiction. Admit that $p<k$. From $\beta_{k, m}=\gamma_{n} \beta_{p, q}$ it follows that $\beta_{p, q}=\gamma_{n} \beta_{k, m}$ and so $p=n-(k+3 m)+1$. This provides again $n<n$, as in the previous case. Suppose now that $p=k$. Then $q \neq m$ and we have $p=n-(p+3 m)+1 \neq n-(p+3 q)+1=k$, i.e. $p \neq k$, a contradiction. This allows us to conclude that $\delta_{k, m} \neq \delta_{p, q}$, whenever $k, m, p, q \in\left\{2, \ldots, \frac{n-1}{2}\right\}$, with $2 k+3 m, 2 p+3 q \leq n+1$ and $k \neq p$ or $m \neq q$. Thus $\left|B^{(6)}\right|=\sum_{k=2}^{\frac{n-5}{2}}\left(\left\lfloor\frac{n+1-2 k}{3}\right\rfloor-1\right)$. 
Take $A^{(6)}=A^{(5)} \cup B^{(6)}$. Since $A^{(5)} \cap B^{(6)}=\emptyset$, we obtain

$$
\left|A^{(6)}\right|=\left|A^{(5)}\right|+\left|B^{(6)}\right|=\frac{3}{2}(n-1)+\sum_{k=2}^{\frac{n-5}{2}}\left(\left\lfloor\frac{n+1-2 k}{3}\right\rfloor-1\right)=\left|G_{n}\right| .
$$

Since $A^{(6)} \subseteq A$, we have $|A| \geq\left|A^{(6)}\right|=\frac{3}{2}(n-1)+\sum_{k=2}^{\frac{n-5}{2}}\left(\left\lfloor\frac{n+1-2 k}{3}\right\rfloor-1\right)$, which allows us to deduce that $\operatorname{rank}\left(\mathcal{T F}_{n}\right) \geq \frac{3}{2}(n-1)+\sum_{k=2}^{\frac{n-5}{2}}\left(\left\lfloor\frac{n+1-2 k}{3}\right\rfloor-1\right)=\left|G_{n}\right|$, as required.

Next, we consider the even case.

Lemma 3.6. Let $n$ be an even number. Then $\operatorname{rank}\left(\mathcal{T F}_{n}\right) \geq 3 n-8+\sum_{k=2}^{n-7}\left(\left\lfloor\frac{n-1-k}{3}\right\rfloor-1\right)=\left|G_{n}\right|$.

Proof. Let $A$ be a generating set of $\mathcal{T F}_{n}$.

Since $\left\{\alpha \in \mathcal{T F}_{n} \mid \operatorname{rank} \alpha=n\right\}=\left\{\operatorname{id}_{n}\right\}$, we have $\operatorname{id}_{n} \in A$. Let $A^{(0)}=\left\{\operatorname{id}_{n}\right\}$. Then $\left|A^{(0)}\right|=1$.

Let $\alpha \in \mathcal{T F}_{n}$ be such that rank $\alpha \leq n-1$. Then, there exist $\alpha_{1}, \ldots, \alpha_{p} \in A \backslash\left\{\operatorname{id}_{n}\right\}$ such that $\alpha=\alpha_{1} \ldots \alpha_{p}$, for some natural number $p$. Clearly, $\operatorname{Ker} \alpha_{1} \subseteq \operatorname{Ker} \alpha$ and $\operatorname{rank} \alpha_{1} \leq n-1$.

If $\alpha \in B^{(1)}=\left\{\alpha_{1,2}, \alpha_{1,3}, \alpha_{n-1, n}, \alpha_{n-2, n}\right\}$ then it is easy to verify that $\alpha=\alpha_{1}$. Hence $B^{(1)} \subseteq A$ and we define $A^{(1)}=A^{(0)} \cup B^{(1)}$. We have $\left|A^{(1)}\right|=\left|A^{(0)}\right|+\left|B^{(1)}\right|=1+4=5$.

Let $\alpha=\alpha_{k, k+2}$, for some $2 \leq k \leq n-4$. Then $(k, k+2) \in \operatorname{Ker} \alpha_{1}$ or $(k+1, k+3) \in \operatorname{Ker} \alpha_{1}$. Since $2 \leq k<n-3$, we have $\operatorname{Rel}(\{k, k+2\})=\{k-1, k+1, k+3\} \subseteq \bar{n}$ or $\operatorname{Rel}(\{k+1, k+3\})=\{k, k+2, k+4\} \subseteq \bar{n}$, respectively. Since Ker $\alpha_{1} \subseteq \operatorname{Ker} \alpha_{k, k+2}$, we obtain Ker $\alpha_{1}=\operatorname{Ker} \alpha_{k, k+2}$. Hence, there exists $\rho_{k, k+2} \in A$ such that $\operatorname{Ker} \rho_{k, k+2}=\operatorname{Ker} \alpha_{k, k+2}$. Thus, being

$$
B^{(2)}=\left\{\rho_{k, k+2} \mid k \in\{2, \ldots, n-4\}\right\},
$$

we have $\left|B^{(2)}\right|=n-5$. Take $A^{(2)}=A^{(1)} \cup B^{(2)}$. Since rank $\rho_{k, k+2}=n-2$, it follows that $\rho_{k, k+2} \notin A^{(1)}$. Then $\left|A^{(2)}\right|=\left|A^{(1)}\right|+\left|B^{(2)}\right|=5+n-5=n$.

Let $\alpha=\alpha_{k, k+1, k+2}^{e}$, for some $k \in\{2, \ldots, n-3\}$. Then there is no $\beta \in \mathcal{T F}_{n}$ such that $\operatorname{rank} \beta=n-1$ and $\operatorname{Ker} \beta \subseteq \operatorname{Ker} \alpha_{k, k+1, k+2}^{e}$. Thus, there exists $\rho_{k, k+1, k+2} \in A$ with $\operatorname{Ker} \rho_{k, k+1, k+2}=\operatorname{Ker} \alpha_{k, k+1, k+2}^{e}$. Clearly, $\rho_{k, k+1, k+2} \notin A^{(2)}$. Take

$$
B^{(3)}=\left\{\rho_{k, k+1, k+2} \mid k \in\{2, \ldots, n-3\}\right\} .
$$

Then $\left|B^{(3)}\right|=n-4$. Furthermore, being $A^{(3)}=A^{(2)} \cup B^{(3)}$, we have $\left|A^{(3)}\right|=\left|A^{(2)}\right|+\left|B^{(3)}\right|=n+n-4=2 n-4$.

Let $\alpha=\alpha_{1,2 k+1}$, for some $k \in\left\{2, \ldots, \frac{n}{2}-1\right\}$. It is clear that

$$
\operatorname{Ker} \alpha_{1,2 k+1}=\{(1+i, 2 k+1-i): 0 \leq i \leq k-1\} \cup\{(x, x): x \in \bar{n}\} .
$$

If $i \in\{1, \ldots, k-2\}$ is such that $(1+i, 2 k+1-i) \in \operatorname{Ker} \alpha_{1}$ then

$$
\operatorname{Rel}(\{1+i, 2 k+1-i\})=\{1+i-1,2 k+1-i-1,1+i+1,2 k+1-i+1\}
$$

and, as $\operatorname{Ker} \alpha_{1} \subseteq \operatorname{Ker} \alpha_{1,2 k+1}$, it follows $(1+(i+1), 2 k+1-(i+1)) \in \operatorname{Ker} \alpha_{1}$ and $(1+(i-1), 2 k+1-(i-1)) \in \operatorname{Ker} \alpha_{1}$. If $(k, k+2) \in \operatorname{Ker} \alpha_{1}$ then $\operatorname{Rel}(\{k, k+2\})=\{k-1, k+1, k+3\}$, whence $(k-1, k+3) \in \operatorname{Ker} \alpha_{1}$ (since $\left.\operatorname{Ker} \alpha_{1} \subseteq \operatorname{Ker} \alpha_{1,2 k+1}\right)$. If $(1,2 k+1) \in \operatorname{Ker} \alpha_{1}$ then $\operatorname{Rel}(\{1,2 k+1\})=\{2,2 k, 2 k+2\} \subseteq \bar{n}\left(\right.$ note that $k \leq \frac{n}{2}-1$ implies $2 k+2 \leq n)$ and, since $\operatorname{Ker} \alpha_{1} \subseteq \operatorname{Ker} \alpha_{1,2 k+1}$, we have $(2,2 k) \in \operatorname{Ker} \alpha_{1}$. Therefore $\operatorname{Ker} \alpha_{1}=\operatorname{Ker} \alpha_{1,2 k+1}$ and there exists $\rho_{1,2 k+1} \in A$ with $\operatorname{Ker} \rho_{1,2 k+1}=\operatorname{Ker} \alpha_{1,2 k+1}$. Clearly, $\rho_{1,2 k+1} \notin A^{(3)}$.

Let $\alpha=\alpha_{2 m, n}$, for some $m \in\left\{1, \ldots, \frac{n-4}{2}\right\}$. Analogously, we can show there exists $\rho_{2 m, n} \in A$ with Ker $\rho_{2 m, n}=$ $\operatorname{Ker} \alpha_{2 m, n}$. Moreover, it is easy to verify that $\rho_{2 m, n} \notin A^{(3)}$ and $\rho_{2 m, n} \neq \rho_{1,2 k+1}$, since $(2 m, n) \in \operatorname{Ker} \rho_{2 m, n}$ and $(2 m, n) \notin \operatorname{Ker} \rho_{1,2 k+1}$, for $k \in\left\{2, \ldots, \frac{n}{2}-1\right\}$. 
Take

$$
B^{(4)}=\left\{\rho_{1,2 k+1} \mid k \in\left\{2, \ldots, \frac{n}{2}-1\right\}\right\} \cup\left\{\rho_{2 m, n} \mid m \in\left\{1, \ldots, \frac{n-4}{2}\right\}\right\} .
$$

Then $\left|B^{(4)}\right|=\frac{n-4}{2}+\frac{n-4}{2}=n-4$. Furthermore, define $A^{(4)}=A^{(3)} \cup B^{(4)}$. Since $A^{(3)} \cap B^{(4)}=\emptyset$, it follows that $\left|A^{(4)}\right|=\left|A^{(3)}\right|+\left|B^{(4)}\right|=2 n-4+n-4=3 n-8$.

Let $\alpha=\beta_{k, m}$, for some $k, m \in\{2, \ldots, n\}$ such that $k+3 m \leq n-1$. Similarly to the proof of Lemma 3.5, we can prove the existence of an element $\delta_{k, m} \in A$ such that $\operatorname{Ker} \delta_{k, m}=\operatorname{Ker} \beta_{k, m}$. Clearly, we also have $\delta_{k, m} \notin A^{(4)}$. Take

$$
B^{(5)}=\left\{\delta_{k, m} \mid k, m \in\{2, \ldots, n\} \text { and } k+3 m \leq n-1\right\}
$$

Then $\left|B^{(5)}\right|=\sum_{k=2}^{n-7}\left(\left\lfloor\frac{n-1-k}{3}\right\rfloor-1\right)$. Moreover, being $A^{(5)}=A^{(4)} \cup B^{(5)}$, since $A^{(4)} \cap B^{(5)}=\emptyset$, we obtain

$$
\left|A^{(5)}\right|=\left|A^{(4)}\right|+\left|B^{(5)}\right|=3 n-8+\sum_{k=2}^{n-7}\left(\left\lfloor\frac{n-1-k}{3}\right\rfloor-1\right)=\left|G_{n}\right| .
$$

Since $A^{(5)} \subseteq A$, we have $|A| \geq\left|A^{(5)}\right|=3 n-8+\sum_{k=2}^{n-7}\left(\left\lfloor\frac{n-1-k}{3}\right\rfloor-1\right)$, which allows us to conclude that $\operatorname{rank}\left(\mathcal{T F}_{n}\right) \geq$ $3 n-8+\sum_{k=2}^{n-7}\left(\left\lfloor\frac{n-1-k}{3}\right\rfloor-1\right)=\left|G_{n}\right|$, as required.

As an immediate consequence of Proposition 3.4 and Lemmas 3.5 and 3.6, we can state our main result.

Theorem 3.7. We have

$$
\operatorname{rank}\left(\mathcal{T F}_{n}\right)= \begin{cases}\frac{3}{2}(n-1)+\sum_{k=2}^{\frac{n-5}{2}}\left(\left\lfloor\frac{n+1-2 k}{3}\right\rfloor-1\right) & \text { if } n \text { is odd } \\ 3 n-8+\sum_{k=2}^{n-7}\left(\left\lfloor\frac{n-1-k}{3}\right\rfloor-1\right) & \text { if } n \text { is even. }\end{cases}
$$

\section{Acknowledgement}

This work was produced during the visit of the second and third authors to CMA, FCT NOVA, Lisbon in January/February 2017. The second author was supported by CMA through a visiting researcher fellowship.

\section{References}

[1] A.Ya. Aüzenštat, The defining relations of the endomorphism semigroup of a finite linearly ordered set, Sibirsk. Mat. 3 (1962), 161-169 (Russian).

[2] A.Ya. Aǔzenštat, Homomorphisms of semigroups of endomorphisms of ordered sets, Uch. Zap., Leningr. Gos. Pedagog. Inst. 238 (1962), 38-48 (Russian).

[3] J. Almeida and M.V. Volkov, The gap between partial and full, Int. J. Algebra Comput. 8 (1998), 399-430.

[4] R. Chinram, R. Srithus and R. Tanyawong, Regular subsemigroups of the semigroups of transformations preserving a fence, Asian-European Journal of Mathematics 9 (2016), 1650003.

[5] J.D. Currie and T.I. Visentin, The number of order-preserving maps of fences and crowns, Order 8 (1991), $133-142$.

[6] I. Dimitrova and J. Koppitz, On the semigroup of all partial fence preserving injections on a finite set, Journal of algebra and its applications 16 (11) (2017), 1750223. 
[7] V.H. Fernandes, Semigroups of order-preserving mappings on a finite chain: a new class of divisors, Semigroup Forum 54 (1997), 230-236.

[8] V.H. Fernandes, Semigroups of order-preserving mappings on a finite chain: another class of divisors, Izv. Vyssh. Uchebn. Zaved. Mat., 2002, Number 3, 51-59 (Russian).

[9] V.H. Fernandes, M.M. Jesus, V. Maltcev and J.D. Mitchell, Endomorphisms of the semigroup of orderpreserving mappings, Semigroup Forum 81 (2010), 277-285.

[10] V.H. Fernandes and M.V. Volkov, On divisors of semigroups of order-preserving mappings of a finite chain, Semigroup Forum 81 (2010), 551-554.

[11] G.M.S. Gomes and J.M. Howie, On the ranks of certain semigroups of order-preserving transformations, Semigroup Forum 45 (1992), 272-282.

[12] P.M. Higgins, Divisors of semigroups of order-preserving mappings on a finite chain, Int. J. Algebra Comput. 5 (1995), 725-742.

[13] J.M. Howie, Products of idempotents in certain semigroups of transformations, Proc. Edinburgh Math. Soc. 17 (2) (1971), 223-236.

[14] J.M. Howie, Fundamentals of Semigroup Theory, Clarendon Press, Oxford, 1995.

[15] K. Jendana and R. Srithus, Coregularity of order-preserving self-mapping semigroups of fences, Commun. Korean Math. Soc. 30 (2015), 349-361.

[16] V.B. Repnitskiı̌ and A. Vernitskii, Semigroups of order preserving mappings, Comm. in Algebra 28 (8) (2000), 3635-3641.

[17] V.B. Repnitskiı̌ and M.V. Volkov, The finite basis problem for the pseudovariety $\mathcal{O}$, Proc. R. Soc. Edinb., Sect. A, Math. 128 (1998), 661-669.

[18] A. Rutkowski, The formula for the number of order-preserving self-mappings of a fence, Order 9 (2) (1992), $127-137$.

[19] A. Vernitskii and M.V. Volkov, A proof and generalisation of Higgins' division theorem for semigroups of order-preserving mappings, Izv. Vyssh. Uchebn. Zaved. Mat., Number 1 (1995), 38-44 (Russian).

Vítor H. Fernandes, CMA, Departamento de Matemática, Faculdade de Ciências e Tecnologia, Universidade NOVA de Lisboa, Monte da Caparica, 2829-516 Caparica, Portugal; e-mail: vhf@fct.unl.pt.

JÖRG KopPITZ, Institute of Mathematics, University of Potsdam, 14476 Potsdam, Germany; e-mail: koppitz@uni-potsdam.de. Also: Institute of Mathematics and Informatics Bulgarian Academy of Sciences, Acad. G. Bonchev Str. bl. 81113 Sofia, Bulgaria; e-mail address: koppitz@math.bas.bg.

Tiwadee Musunthia, Department of Mathematics, Faculty of Science, Silpakorn University, Nakorn Pathom, Thailand 73000; e-mail: tiwadee.m@gmail.com. 